\title{
CORE-COLLAPSE SUPERNOVAE MISSED BY OPTICAL SURVEYS
}

\author{
S. Mattila ${ }^{1}$, T. Dahlen ${ }^{2}$, A. Efstathiou ${ }^{3}$, E. Kankare $^{1}$, J. Melinder $^{4}$, A. Alonso-Herrero ${ }^{5}$,

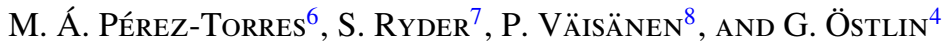 \\ ${ }^{1}$ Tuorla Observatory, Department of Physics and Astronomy, University of Turku, Väisäläntie 20, FI-21500 Piikkiö, Finland; sepmat@utu.fi \\ ${ }^{2}$ Space Telescope Science Institute, 3700 San Martin Drive, Baltimore, MD 21218, USA \\ ${ }^{3}$ School of Sciences, European University Cyprus, Diogenes Street, Engomi, 1516 Nicosia, Cyprus \\ ${ }^{4}$ Department of Astronomy, Oskar Klein Centre, Stockholm University, AlbaNova University Centre, SE-106 91 Stockholm, Sweden \\ ${ }^{5}$ Instituto de Fisica de Cantabria, CSIC-UC, Avenida de los Castros s/n, E-39005 Santander, Spain \\ ${ }^{6}$ Instituto de Astrofísica de Andalucía-CSIC, P.O. Box 3004, E-18008 Granada, Spain \\ ${ }^{7}$ Australian Astronomical Observatory, P.O. Box 296, Epping, NSW 1710, Australia \\ ${ }^{8}$ South African Astronomical Observatory, P.O. Box 9, Observatory 7935, Cape Town, South Africa \\ Received 2012 April 5; accepted 2012 June 19; published 2012 August 21
}

\begin{abstract}
We estimate the fraction of core-collapse supernovae (CCSNe) that remain undetected by optical SN searches due to obscuration by large amounts of dust in their host galaxies. This effect is especially important in luminous and ultraluminous infrared galaxies, which are locally rare but dominate the star formation at redshifts of $z \sim 1-2$. We perform a detailed investigation of the SN activity in the nearby luminous infrared galaxy Arp 299 and estimate that up to $83 \%$ of the SNe in Arp 299 and in similar galaxies in the local universe are missed by observations at optical wavelengths. For rest-frame optical surveys we find the fraction of SNe missed due to high dust extinction to increase from the average local value of $\sim 19 \%$ to $\sim 38 \%$ at $z \sim 1.2$ and then remain roughly constant up to $z \sim 2$. It is therefore crucial to take into account the effects of obscuration by dust when determining SN rates at high redshift and when predicting the number of CCSNe detectable by future high- $z$ surveys such as LSST, JWST, and Euclid. For a sample of nearby CCSNe (distances 6-15 Mpc) detected during the last $12 \mathrm{yr}$, we find a lower limit for the local CCSN rate of $1.5_{-0.3}^{+0.4} \times 10^{-4} \mathrm{yr}^{-1} \mathrm{Mpc}^{-3}$, consistent with that expected from the star formation rate. Even closer, at distances less than $\sim 6 \mathrm{Mpc}$, we find a significant increase in the CCSN rate, indicating a local overdensity of star formation caused by a small number of galaxies that have each hosted multiple SNe.
\end{abstract}

Key words: galaxies: individual (Arp 299) - galaxies: starburst - infrared: galaxies - supernovae: general supernovae: individual (SN 2005at, SN 2010P)

Online-only material: color figures

\section{INTRODUCTION}

Much of the massive star formation and hence a substantial fraction of the core-collapse supernovae (CCSNe) in the universe may be hidden behind dust. At higher redshifts, obscured star formation in luminous $\left(10^{11} L_{\odot} \leqslant L_{\mathrm{IR}}<10^{12} L_{\odot}\right)$ and ultraluminous $\left(L_{\mathrm{IR}}>10^{12} L_{\odot}\right)$ infrared (IR) galaxies (LIRGs and ULIRGs, respectively) actually dominates over the star formation seen in the ultraviolet (UV) and optical (e.g., Le Floc'h et al. 2005; Magnelli et al. 2009, 2011). Because of the observed concentration of star formation within the innermost nuclear regions (e.g., Soifer et al. 2001), high spatial resolution is crucial for detecting $\mathrm{SNe}$ in these environments. This can be achieved with observations from space, with ground-based adaptive optics (AO) imaging observations at near-IR wavelengths, or with interferometric radio imaging. High spatial resolution searches at nearIR wavelengths have already discovered several obscured $\mathrm{SNe}$ within a few hundred parsecs from LIRG nuclei (Mattila et al. 2007; Kankare et al. 2008, 2012). Furthermore, high spatial resolution searches at radio wavelengths have been able to reveal $\mathrm{SN}$ factories within the innermost $\sim 100$ pc LIRG nuclear regions that have so far remained hidden at all other wavelengths (e.g., Lonsdale et al. 2006; Pérez-Torres et al. 2009; Ulvestad 2009; Romero-Cañizales et al. 2011, 2012; Bondi et al. 2012; Herrero-Illana et al. 2012).

The effects of host galaxy extinction on the detectability of $\mathrm{SNe}$ are expected to increase with redshift since, in general, shorter rest-frame wavelengths are observed at higher redshifts.
More importantly, the fraction of the star formation hidden from optical searches in LIRGs and ULIRGs is expected to increase rapidly toward redshift $z \sim 1$ (Pérez-González et al. 2005; Le Floc'h et al. 2005; Caputi et al. 2007; Magnelli et al. 2009, 2011). Unless properly corrected for, errors in derived CCSN rates at $z \sim 1$ will be dominated by these effects (Mannucci et al. 2007; Dahlen et al. 2012; Melinder et al. 2012). Recent CCSN rate studies (e.g., Dahlen et al. 2004; Botticella et al. 2008; Bazin et al. 2009; Graur et al. 2011; Li et al. 2011a; Horiuchi et al. 2011) have indicated that the cosmic CCSN rate might not match the massive star formation rate (SFR) even in the local universe. This could be caused by a population of SNe remaining undetected by the current optical searches either because they are intrinsically faint or dark due to large host galaxy extinctions (e.g., Horiuchi et al. 2011). In addition, $\mathrm{SNe}$ with lower extinctions but occurring within a few hundred parsecs of an LIRG nucleus would likely be detectable only by observations with a high spatial resolution (e.g., Kankare et al. 2012) typically not available for the current SN searches. More recently, Botticella et al. (2012) used a sample of 14 CCSNe within the $11 \mathrm{Mpc}$ volume to derive a robust lower limit for the local CCSN rate. They found the volumetric CCSN rate to be consistent with that expected from the SFR derived from far-UV luminosities and higher than expected based on $\mathrm{H} \alpha$ luminosities. This indicates that most of the intrinsically faint and/or dark events were detected in their local sample.

The fraction of missing $\mathrm{SNe}$ as a function of redshift has been studied previously by Mannucci et al. (2007). They compiled the 
star formation densities for different redshifts derived from UV and IR observations. They used these results together with their own estimates on how many $\mathrm{SNe}$ are lost due to obscuration by dust in local starburst galaxies, LIRGs, and ULIRGs to derive a correction for $\mathrm{SN}$ rates at high redshifts. These estimates, however, were based on a very small number of SNe detected in such galaxies by that time (Maiolino et al. 2002; Mannucci et al. 2003). Also, at that time, very little was known about the nature of the high-redshift LIRGs and ULIRGs. Mannucci et al. (2007) assumed that they were the same kind of systems as in the local universe, which was not an unreasonable assumption. However, later developments, in particular the recent results from Spitzer and Herschel, have shown that the high-redshift $\mathrm{U} / \mathrm{LIRG}$ population is dominated by disk galaxies forming stars in the "normal" extended (the so-called main sequence) mode rather than in compact starbursts as observed in the local U/ LIRGs (e.g., Elbaz et al. 2011).

In this investigation we use a somewhat similar approach to that of Mannucci et al. (2007) to estimate the corrections needed in order to account for CCSNe remaining undetected by optical surveys both locally and as a function of redshift. Our corrections consist of two parts. First, we estimate the fraction of CCSNe in normal galaxies with substantially higher host galaxy extinctions than predicted by simple models for the smooth dust distribution and the resulting inclination effects. Thereafter, we estimate the fraction of CCSNe missed in local U/LIRGs. For this we make use of the rich SN population of one of the nearest LIRGs, Arp 299. Assuming that the SNe with the highest host galaxy extinctions are missed by the optical searches and not compensated for by the standard extinction corrections, we can derive the fraction of $\mathrm{SNe}$ that remain missing and estimate the corrections needed to be applied when deriving CCSN rates. We then use this information together with the latest knowledge of the nature and evolution of high- $z$ U/LIRGs to calculate the fraction of CCSNe missed as a function of redshift. We assume $H_{0}=70 \mathrm{~km} \mathrm{~s}^{-1} \mathrm{Mpc}^{-1}, \Omega_{\Lambda}=0.7$, and $\Omega_{M}=0.3$ throughout the paper.

\section{SUPERNOVA BUDGET OF NEARBY GALAXIES}

\subsection{The Nearby Supernova Sample}

In order to investigate the completeness of the current local optical SN searches at different distances, we selected all the SNe discovered by the end of 2011 and after the beginning of the year 2000 from the Asiago SN catalog (Barbon et al. 1999) with an identified host galaxy and $v_{\text {rec }}<1500 \mathrm{~km} \mathrm{~s}^{-1}$ (corresponding to distances less than $\sim 20 \mathrm{Mpc}$ ). We then adopted their radial velocities corrected for the peculiar velocities due to Virgo infall from the HyperLeda database (Paturel et al. 2003) and converted to distances. We ended up with a sample of 100 objects including also a few events without a spectroscopic classification, Type Ia's and luminous blue variable (LBV) outbursts originally classified as $\mathrm{SNe}$. We then investigated the volumetric rate of these transients within different distances over the $12 \mathrm{yr}$ period. A significantly higher rate was found at the smallest distances of $\sim 4-6 \mathrm{Mpc}$ indicative of the local overdensity of star formation (see the discussion below). The rate was found to stay roughly constant between $\sim 12$ and $\sim 15 \mathrm{Mpc}$ and then show a significant decline. The drop of the rate at distances greater than $\sim 15 \mathrm{Mpc}$ could be a result of the SN searches starting to miss a significant fraction of both the intrinsically faint and heavily dust-obscured events after this point (see also Botticella et al. 2012 and Horiuchi et al. 2011).
In the following analysis we therefore only consider the 49 events with Virgo infall corrected recession velocities of less than $1050 \mathrm{~km} \mathrm{~s}^{-1}$ (or distances less than $15 \mathrm{Mpc}$ ). We excluded any Type Ia SNe (SNe 2001el, 2005df, 2005ew, 2006E, 2006mq, 2008ge, 2010ae, and 2011fe) and LBV outbursts (SNe 2000ch, 2002kg, and 2010da; Wagner et al. 2004; Pastorello et al. 2010; Smith et al. 2011; Maund et al. 2006). The initial sample also includes one event, SN 2008eh, without a spectroscopic classification. Based on its discovery magnitude and some light curve information, Horiuchi et al. (2011) assumed that it was an intrinsically faint CCSN. However, without any certain information on the nature of this event, we decide to exclude it from our final sample. Two of the remaining events with estimated distances larger than $15 \mathrm{Mpc}$ were also excluded from our analysis. SN 2003hn occurred in NGC 1448, which also hosted the well-observed normal Type Ia SN 2001el. The optical and near-IR photometry of SN 2001el yielded a distance of 17.9 Mpc for NGC 1448 (Krisciunas et al. 2003; Mattila et al. 2005b). The host galaxy of SN 2004gk with a negative (Virgo infall corrected) recession velocity has a Tully-Fisher based distance of $\sim 20 \mathrm{Mpc}$ (Solanes et al. 2002). We are therefore left with a total of 35 events which are listed in Table 1.

We also initially included SN 2008S-like events as a part of the analysis. We have therefore added the NGC 300-2008OT in Table 1, which is an event similar to SN 2008S and missing from the Asiago catalog. In total, our initial sample includes four such events: SNe 2002bu, 2008S, 2010dn, and NGC 3002008OT. A number of authors (e.g., Botticella et al. 2009; Pumo et al. 2009) favor a scenario where the SN 2008 S-like events originate from an explosion of an extreme asymptotic giant branch star as an electron capture SN. However, several others found a non-explosive outburst of a massive star the most plausible origin (e.g., Bond et al. 2009; Berger et al. 2009; Smith et al. 2009; Kashi et al. 2010). Recent optical observations of the SN 2008S site (Szczygiel et al. 2012) have already ruled out the presence of a massive evolved progenitor star indicating that either it did not survive the 2008 event or has now returned to its dust enshrouded state. Kochanek (2011) predicts that the shock powering the current IR luminosity from the site of SN 2008S should destroy the dust, eventually allowing a direct confirmation if the progenitor of SN 2008S has disappeared. With the origin of the SN 2008S-like events still an open question, we decided to exclude them from our current analysis. We note that the SN 2008S-like events were also excluded by Botticella et al. (2012) from their CCSN rate analysis.

Our sample also includes the recently reported SN 2008jb that remained undetected by all the pointed SN searches despite a distance of only $\sim 9 \mathrm{Mpc}$ (Prieto et al. 2012). It occurred in a low-luminosity host galaxy, ESO 302-G014, similar to the Magellanic Clouds and not included in the target lists of the pointed SN searches. The fraction of CCSNe occurring in such low-luminosity/metallicity galaxies is very low; $<2 \%$ according to Young et al. (2008). Therefore, such events missed by optical-pointed SN searches locally are not likely to contribute significantly to the fraction of CCSNe missed by optical SN searches in general.

The adopted host galaxy distances are mostly the kinematic distances obtained from the Virgo infall corrected recession velocities. However, for several of the closest galaxies, more accurate distances were available from Cepheid observations or from the tip of the red giant branch (TRGB) method, which were adopted instead. In a few cases, the mean distance from several methods was adopted from the literature. The adopted distances 
Table 1

Volume-limited Sample of SNe Closer Than 15 Mpc Discovered in 2000-2011

\begin{tabular}{|c|c|c|c|c|c|c|c|c|c|c|c|}
\hline $\mathrm{SN}$ & Host Galaxy & $\begin{array}{l}\text { Velocity } \\
\left(\mathrm{km} \mathrm{s}^{-1}\right)\end{array}$ & $\begin{array}{c}\text { Distance } \\
(\mathrm{Mpc})\end{array}$ & Method & Type & $A_{\mathrm{V}}$ Host (Reference) & $\begin{array}{c}A_{\mathrm{V}} \\
\mathrm{MW}\end{array}$ & $M_{\text {peak }}$ & $\begin{array}{c}\text { Incl. } \\
\left({ }^{\circ}\right)\end{array}$ & Botticella & $\begin{array}{c}\log L_{\mathrm{IR}} \\
\left(L_{\odot}\right)\end{array}$ \\
\hline $2000 \mathrm{db}$ & NGC 3949 & 1020 & 14.6 & Kinematic & IIP & & 0.07 & $\cdots$ & 57 & No & 9.93 \\
\hline 2001 ig & NGC 7424 & 754 & 10.8 & Kinematic & IIb & $<0.06^{8}$ (Silverman et al. 2009) & 0.03 & $-17.4^{8}$ & 59 & No & $\ldots$ \\
\hline 2002ap & NGC 628 & 686 & $9.3^{1}$ & Mean & Ic & 0.04 (Takada-Hidai et al. 2002) & 0.24 & $-17.7^{2}$ & 35 & Yes & 9.89 \\
\hline $2002 \mathrm{bu}$ & NGC 4242 & 737 & 10.5 & Kinematic & 08S-like & $\ldots$ & 0.04 & $\ldots$ & 52 & Yes & $\ldots$ \\
\hline $2002 \mathrm{hh}$ & NGC 6946 & 318 & $5.7^{2}$ & Mean & IIP & 4.1 (Pozzo et al. 2006) & 1.13 & $-18.4^{2}$ & 31 & Yes & 9.94 \\
\hline $2003 \mathrm{~J}$ & NGC 4157 & 1011 & 14.4 & Kinematic & IIP & $\ldots$ & 0.07 & $\ldots$ & 90 & No & 10.19 \\
\hline $2003 \mathrm{gd}$ & NGC 628 & 686 & $9.3^{1}$ & Mean & IIP & 0.2 (Smartt et al. 2009) & 0.23 & $-16.6^{2}$ & 35 & Yes & 9.89 \\
\hline 2003ie & NGC 4051 & 917 & 13.1 & Kinematic & IIP & $\ldots$ & 0.04 & $\ldots$ & 30 & No & 9.90 \\
\hline 2003jg & NGC 2997 & 914 & 13.1 & Kinematic & $\mathrm{Ib} / \mathrm{c}$ & 3.7 N. Elias-Rosa et al. (in preparation) & 0.36 & $-17.8^{8}$ & 32 & No & $\ldots$ \\
\hline $2004 \mathrm{am}$ & M 82 & 487 & $3.3^{3}$ & Cepheid & IIP & ～5 (Mattila et al. 2012) & 0.53 & $-15.4^{12}$ & 79 & Yes & 10.66 \\
\hline $2004 d j$ & NGC 2403 & 370 & $3.3^{3}$ & Cepheid & IIP & 0.4 (Smartt et al. 2009) & 0.13 & $-16.6^{2}$ & 60 & Yes & 9.21 \\
\hline 2004 et & NGC 6946 & 318 & $5.7^{2}$ & Mean & IIP & 0.2 (Smartt et al. 2009) & 1.13 & $-17.9^{2}$ & 31 & Yes & 9.94 \\
\hline $2005 \mathrm{ae}$ & ESO 209-G009 & 862 & 12.3 & Kinematic & $\mathrm{IIb}$ & $\ldots$ & 0.86 & $\ldots$ & 90 & No & 9.85 \\
\hline 2005af & NGC 4945 & 376 & $3.8^{4}$ & TRGB & IIP & $\sim 0$ (Pereyra et al. 2006) & 0.61 & $-15.7^{2}$ & 90 & Yes & 10.45 \\
\hline 2005 at & NGC 6744 & 618 & 8.8 & Kinematic & Ic & $2.3 \pm 0.3($ E. Kankare et al. in preparation $)$ & 0.14 & $-16.1^{2}$ & 54 & Yes & 9.89 \\
\hline 2005ay & NGC 3938 & 1017 & 14.5 & Kinematic & IIP & $\ldots$ & 0.07 & $\ldots$ & 14 & No & 9.92 \\
\hline $2005 \mathrm{cs}$ & M 51 & 702 & 10.0 & Kinematic & IIP & 0.3 (Smartt et al. 2009) & 0.12 & $-15.9^{2}$ & 30 & Yes & $\ldots$ \\
\hline 2006my & NGC 4651 & 912 & 13.0 & Kinematic & II & $\ldots$ & 0.09 & $\ldots$ & 50 & No & 9.58 \\
\hline $2007 \mathrm{gr}$ & NGC 1058 & 634 & $9.3^{5}$ & Cepheid & Ic & 0.09 (Hunter et al. 2009) & 0.21 & $-17.5^{2}$ & 20 & Yes & $\ldots$ \\
\hline 2007it & NGC 5530 & 1046 & 14.9 & Kinematic & II & $\ldots$ & 0.39 & $\ldots$ & 67 & No & $\ldots$ \\
\hline $2008 S$ & NGC 6946 & 318 & $5.7^{2}$ & Mean & 08S-like & $\ldots$ & 1.12 & $\ldots$ & 31 & No & 9.94 \\
\hline N300-OT & NGC 300 & -38 & $1.9^{6}$ & Cepheid & 08S-like & $\ldots$ & 0.04 & $\ldots$ & 40 & No & 8.35 \\
\hline 2008ax & NGC 4490 & 797 & 11.4 & Kinematic & $\mathrm{IIb}$ & $1.5($ Chornock et al. 2011) & 0.07 & $-18.5^{2}$ & 47 & Yes & 10.28 \\
\hline $2008 \mathrm{bk}$ & NGC 7793 & 60 & $3.4^{7}$ & Cepheid & IIP & $\sim 0$ (Van Dyk et al. 2012) & 0.07 & $-15.2^{2}$ & 53 & Yes & 8.92 \\
\hline $2008 \mathrm{iz}$ & M 82 & 487 & $3.3^{3}$ & Cepheid & $\mathrm{RSN}$ & $\lesssim 10$ (Mattila et al. 2012) & 0.53 & $\ldots$ & 79 & No & 10.66 \\
\hline 2008jb & ESO 302-G014 & 636 & 9.1 & Kinematic & IIP & 0.19 (Prieto et al. 2012) & 0.03 & $-15.3^{9}$ & 74 & No & $\ldots$ \\
\hline $2009 N$ & NGC 4487 & 1026 & 14.7 & Kinematic & IIP & $\ldots$ & 0.07 & $\ldots$ & 46 & No & $\ldots$ \\
\hline 2009dd & NGC 4088 & 989 & 14.1 & Kinematic & II & $\ldots$ & 0.07 & $\ldots$ & 71 & No & 10.29 \\
\hline 2009hd & NGC 3627 & 788 & $9.4^{3}$ & Cepheid & II & 3.7 (Elias-Rosa et al. 2011) & 0.11 & $-17.7^{2}$ & 57 & Yes & 10.33 \\
\hline 2009ib & NGC 1559 & 1004 & 14.3 & Kinematic & IIP & $\ldots$ & 0.10 & $\ldots$ & 60 & No & 10.31 \\
\hline 20091s & NGC 3423 & 1032 & 14.7 & Kinematic & II & $\cdots$ & 0.10 & $\cdots$ & 32 & No & $\cdots$ \\
\hline $2010 \mathrm{br}$ & NGC 4051 & 917 & 13.1 & Kinematic & $\mathrm{Ib} / \mathrm{c}$ & $\ldots$ & 0.04 & $\ldots$ & 30 & No & 9.90 \\
\hline 2010dn & NGC 3184 & 766 & 10.9 & Kinematic & 08S-like & $\ldots$ & 0.06 & $\ldots$ & 24 & No & 9.67 \\
\hline $2011 \mathrm{dh}$ & M 51 & 702 & 10.0 & Kinematic & $\mathrm{IIb}$ & $<0.15$ (Arcavi et al. 2011) & 0.12 & $-17.0^{10}$ & 30 & No & $\ldots$ \\
\hline $2011 \mathrm{ja}$ & NGC 4945 & 376 & $3.8^{4}$ & TRGB & IIP & $>3($ Monard et al. 2011) & 0.59 & $-17.5^{11}$ & 90 & No & 10.45 \\
\hline $2011 \mathrm{jm}$ & NGC 4809 & 979 & 14.0 & Kinematic & Ic & $\ldots$ & 0.11 & $\ldots$ & 90 & No & $\ldots$ \\
\hline
\end{tabular}

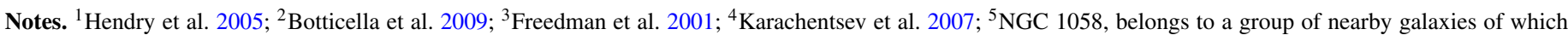

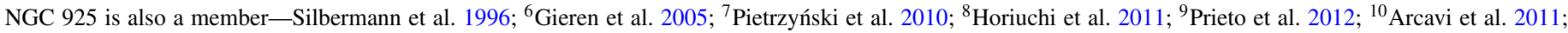
${ }^{11}$ Monard et al. 2011; ${ }^{12}$ Mattila et al. 2012.

are listed in Table 1 with the relevant references given in the notes to the table. In Table 1, we also list the IR luminosities of the host galaxies (if available from Sanders et al. 2003) scaled to our adopted distances.

The volumetric CCSN rates obtained using the events from Table 1 are listed in Table 2. We adopt the small number statistical uncertainties from Gehrels (1986) and use these throughout this study. Excluding the SN 2008S-like events there are a total of eight CCSNe within $6 \mathrm{Mpc}$, and the CCSN rate within $6 \mathrm{Mpc}$ becomes $7.4_{-2.6}^{+3.7} \times 10^{-4} \mathrm{yr}^{-1} \mathrm{Mpc}^{-3}$. This is significantly higher than the volumetric CCSN rate reported by Botticella et al. (2012), and that expected from the SFR extrapolated from high$z$ and even the locally normalized Horiuchi SFR. However, such an elevated CCSN rate is consistent with a local overdensity of star formation observed within $\sim 10 \mathrm{Mpc}$ (e.g., Karachentsev et al. 2004). Furthermore, according to Heckman (1998), only four galaxies (M 82, NGC 253, M 83, and NGC 4945) are responsible for $25 \%$ of all the high-mass $\left(\geqslant 8 M_{\odot}\right)$ star formation within $10 \mathrm{Mpc}$ distance. It is interesting that all of these four galaxies are actually closer than $6 \mathrm{Mpc}$. Of the total of eight
Table 2

Volumetric CCSN Rates Using the Events from Table 1

\begin{tabular}{lcccr}
\hline \hline \multirow{2}{*}{$\begin{array}{l}\text { istance } \\
(\mathrm{Mpc})\end{array}$} & \multicolumn{4}{c}{ SN Rate $\left(10^{-4} \mathrm{yr}^{-1} \mathrm{Mpc}^{-3}\right)$} \\
\cline { 2 - 5 } & \multicolumn{2}{c}{ CCSNe+08S-like } & \multicolumn{2}{c}{ CCSNe } \\
\hline 5 & $11.1(7)$ & $\ldots$ & $9.5(6)$ & $\ldots(0)$ \\
6 & $9.2(10)$ & $\ldots$ & $7.4(8)$ & $\ldots(0)$ \\
7 & $5.8(10)$ & $3.1(2)$ & $4.6(8)$ & $0(0)$ \\
8 & $3.9(10)$ & $1.3(2)$ & $3.1(8)$ & $0(0)$ \\
9 & $3.0(11)$ & $1.2(3)$ & $2.5(9)$ & $0.39(1)$ \\
10 & $3.6(18)$ & $2.5(10)$ & $3.2(16)$ & $2.0(8)$ \\
11 & $3.1(21)$ & $2.3(13)$ & $2.5(17)$ & $1.6(9)$ \\
12 & $2.5(22)$ & $1.8(14)$ & $2.1(18)$ & $1.3(10)$ \\
13 & $2.2(24)$ & $1.6(14)$ & $1.8(20)$ & $1.2(12)$ \\
14 & $2.0(28)$ & $1.6(20)$ & $1.7(24)$ & $1.3(16)$ \\
15 & $2.1(36)$ & $1.8(28)$ & $1.9(32)$ & $1.5(24)$ \\
& & & &
\end{tabular}

Notes. The rates including the entire volume within the given distance are given in Columns 2 and 4, and the rates excluding the volume within $6 \mathrm{Mpc}$ are given in Columns 3 and 5. The number of events included is given in parenthesis. 
Table 3

Comparison between the Observed and Predicted Host Galaxy Extinctions

\begin{tabular}{|c|c|c|c|c|c|c|c|c|c|c|}
\hline \multirow[t]{2}{*}{ Inclination } & \multicolumn{6}{|c|}{ Observed } & \multicolumn{4}{|c|}{ Predicted } \\
\hline & $\left\langle A_{\mathrm{V}}\right\rangle$ & Median & SEM & $\sigma$ & $\mathrm{SNe}$ & Fraction & $\left\langle A_{\mathrm{V}}\right\rangle$ & Median & $\sigma$ & Fraction \\
\hline $0 \leqslant i \leqslant 30$ & 0.18 & 0.15 & 0.06 & 0.11 & 3 & $17 \%$ & 0.37 & 0.15 & 0.52 & $13.5 \%$ \\
\hline $30<i \leqslant 60$ & 1.25 & 0.40 & 0.50 & 1.59 & 10 & $55 \%$ & 0.52 & 0.20 & 0.73 & $36.4 \%$ \\
\hline $60<i \leqslant 90^{\mathrm{a}}$ & 1.06 & 0.19 & 0.97 & 1.68 & 5 & $28 \%$ & 1.89 & 0.57 & 3.58 & $50.1 \%$ \\
\hline \multicolumn{11}{|l|}{ Sample 1} \\
\hline $0 \leqslant i \leqslant 60$ & 1.00 & 0.20 & 0.40 & 1.45 & 13 & $72 \%$ & 0.48 & 0.18 & 0.68 & $49.9 \%$ \\
\hline \multicolumn{11}{|l|}{ Sample $2^{\mathrm{b}}$} \\
\hline $0 \leqslant i \leqslant 60$ & 0.48 & 0.20 & 0.22 & 0.74 & 11 & $72 \%$ & 0.48 & 0.18 & 0.68 & $49.9 \%$ \\
\hline
\end{tabular}

Notes. The predicted distributions follow that of Riello \& Patat (2005) for $B / T=0.0$ and assuming $\tau_{V}(0)=2.5$ adopted to match the observations.

a Excluding SNe 2004am and 2008iz (both being within the nuclear regions of the prototypical starburst galaxy M82) and therefore based on three events.

b Excluding SNe 2002hh and 2009hd with the highest host galaxy extinctions from the sample.

CCSNe within $6 \mathrm{Mpc}$, SNe 2004am and 2008iz occurred in M 82, SNe 2005af and 2011ja in NGC 4945, and SNe 2002hh and 2004et in NGC 6946. Thus, there are only five host galaxies within $6 \mathrm{Mpc}$ that are responsible for all eight events over the last $12 \mathrm{yr}$ within this volume. Also, it is possible that the SFR within $6 \mathrm{Mpc}$ is actually concentrated in more dusty systems such as the prototypical starburst galaxy M 82, and, therefore, the missing fraction of $\mathrm{SNe}$ within this volume might be higher than representative for the local volume.

In order to avoid the effects of overdensity, we calculated the volumetric CCSN rates after excluding the volume within $6 \mathrm{Mpc}$. These rates are listed in Table 2 for different outer limits of the volume. We note that between 10 and $15 \mathrm{Mpc}$ this volumetric CCSN rate stays constant within statistical uncertainties indicating that within $15 \mathrm{Mpc}$ we are not missing a significant number of events. The volumetric CCSN rate at 6-15 Mpc is $1.5_{-03}^{+0.4} \times 10^{-4} \mathrm{yr}^{-1} \mathrm{Mpc}^{-3}$, which is similar to the volumetric CCSN rate recently derived by Botticella et al. (2012) for a similar sample of CCSNe within $11 \mathrm{Mpc}$ (the 14 events used by Botticella et al. are indicated in Column 11 of Table 1). This value is also consistent with the expected CCSN rate from the local star formation density (see Horiuchi et al. 2011).

\subsection{Host Galaxy Extinctions}

For all the SNe within $12 \mathrm{Mpc}$ except SN 2005at, host galaxy extinctions were available in the literature (for references see Column 7 of Table 1). We note that these extinctions were estimated for each SN, not for each galaxy as a whole. Therefore, we concentrate here on the CCSNe within $12 \mathrm{Mpc}$, which is almost identical to the distance limit in the sample of Botticella et al. (2012) when accounting for the difference in the value of $H_{0}$ adopted in the two studies. The Galactic extinctions were adopted from Schlegel et al. (1998). For SN 2005at, which is a Type Ic event resembling SN 1994I (see Schmidt \& Salvo 2005 ) at $\sim 9 \mathrm{Mpc}$, very little information was available in the literature. In E. Kankare et al. (in preparation) we present the available UBVRI photometry of SN 2005at, together with the optical spectrum of Schmidt \& Salvo (2005), covering a wide wavelength range from $\sim 3300$ to $\sim 10200 \AA$. We use these data to estimate a host galaxy extinction of $A_{\mathrm{V}}=2.3 \pm 0.3$ for SN 2005at by comparison with well-observed normal Type Ic events.
In Table 3, we compare the observed extinction properties of our CCSN sample within $12 \mathrm{Mpc}$ with the predictions from our Monte Carlo simulations (E. Kankare et al. in preparation). For this comparison we include the 18 CCSNe from Table 1, excluding the SN 2008S-like events whose origin is still an open question. These simulations follow the recipe in Riello $\&$ Patat (2005), assuming a homogeneous dust distribution for the model host galaxy with the bulge-to-total $(B / T)$ ratio $=$ $0.0(100 \%$ of the SNe located in the disk) and an optical depth through a simulated face-on galaxy at a zero radius of $\tau_{V}(0)=$ 2.5 , consistent with many statistical studies (e.g., Kankare et al. 2009), to derive a distribution of expected $A_{V}$ values. For the purpose of comparing observed and predicted extinction values, we divided our sample into three inclination bins. The host galaxy inclinations were adopted from the HyperLeda database.

First, we compare the observed fraction of CCSNe in different inclination bins with the predictions from the simulations (see Columns 7 and 11 in Table 3). The relative distribution of $\mathrm{SNe}$ in galaxies with inclinations of $0^{\circ}-30^{\circ}$ and $30^{\circ}-60^{\circ}$ appears to be as expected, whereas there is an apparent lack of $\mathrm{SNe}$ in host galaxies with inclinations higher than $60^{\circ}$. Although half of the $\mathrm{SNe}$ would be expected in galaxies with an inclination of $61^{\circ}-90^{\circ}$, less than $30 \%$ were discovered in such galaxies. In the edge-on bin, $\sim 10 \%$ of the SNe have a simulated extinction of $A_{\mathrm{V}}>5$. Therefore, we do expect a fraction of the $\mathrm{SNe}$ to be missed in normal galaxies with the highest inclinations. However, the expected median extinction of objects in the edgeon bin is $\sim 0.6$ in $A_{\mathrm{V}}$, which means that the optical SN searches should have been sensitive to most of the SNe even in the edgeon galaxies within the $12 \mathrm{Mpc}$ volume. Therefore, the apparent lack of events in the edge-on bin appears to be more likely due to the selection effect of the $\mathrm{SN}$ searches avoiding these galaxies rather than being a consequence of the host galaxy extinction. The fact that there is an apparent lack of CCSNe with edge-on host galaxies indicates that our SN sample within $12 \mathrm{Mpc}$ (and the one used by Botticella et al. 2012) is likely not complete. Therefore, the local volumetric CCSN rate could be higher than estimated above, which indicates that the local overdensity of star formation could also affect the results within the 6-15 Mpc distance.

Second, we compare the observed host galaxy extinctions in different inclination bins with the predictions (see Table 3). In the face-on $\left(0^{\circ}-30^{\circ}\right)$ bin the observed average and median extinctions of $A_{\mathrm{V}}=0.18$ and 0.15 , respectively, are quite 
low, similar to the predicted values, although any quantitative comparison between the extinctions is difficult because of the small number of events. However, in the intermediate inclination $\left(30^{\circ}-60^{\circ}\right)$ bin we have $10 \mathrm{SNe}$, allowing for a more meaningful comparison. The average and median extinctions for this sample are 1.25 and 0.40 in $A_{\mathrm{V}}$, which are both significantly higher than their predicted values of 0.52 and 0.20 , respectively, indicating that the smooth distribution of dust used in the simulations does not agree well with the observations. In the edge-on $\left(60^{\circ}-90^{\circ}\right)$ sample we have five events, two of them (SNe 2004am and 2008iz) within the nuclear regions of the prototypical nearby starburst galaxy M 82. SN 2008iz was discovered at radio wavelengths with no reported detection in the optical indicating a very high extinction being within the nuclear regions of M 82 (e.g., Brunthaler et al. 2010). Based on a detection in the near-IR K-band, Mattila et al. (2012) estimated the likely extinction towards SN 2008iz to be not more than $A_{\mathrm{V}} \sim 10$. SN 2004am occurred coincident with the obscured super star cluster M82-L and has an estimated host galaxy extinction of $A_{\mathrm{V}} \sim 5$ (Mattila et al. 2012). Excluding these two events, the average extinction for the remaining three events occurring in normal spiral galaxies is $\sim 1.1$, which is lower than predicted but of course highly uncertain due to the very small number of events included.

\subsection{Intrinsically Faint Supernovae}

Using our adopted distances and extinctions we calculated optical absolute peak magnitudes for the events within a distance of $12 \mathrm{Mpc}$. These are mostly based on the SN peak magnitudes available from Horiuchi et al. (2011) and Botticella et al. (2012). These absolute SN peak magnitudes are very approximate, especially for a couple of the most recent events for which we adopted their discovery magnitudes (for references see the note to Table 1). We find that 3 of the $17 \mathrm{CCSNe}$ within $12 \mathrm{Mpc}$ are fainter than $M=-15.5$, but none of them are fainter than $M=-15.0$. We note that the absolute SN magnitudes considered by Horiuchi et al. (2011) were not corrected for host galaxy extinctions. After applying the correction, we find from their intrinsically faint CCSNe that only SN 2004am has an absolute peak magnitude of $\sim-15$ (see Column 9 in Table 1). These faint $\mathrm{SNe}$ are more likely to be missed in a search over a larger volume compared to the restriction $<12 \mathrm{Mpc}$ for our sample and could therefore lead to underestimates of the CCSN rate. The absolute peak magnitudes listed in Table 1 (excluding SN 2008iz with no reported optical detection) therefore suggest that a correction factor approximately $\sim 1.2$ may actually be needed to account for the intrinsically faint CCSNe in a survey that is not deep enough for their detection. However, such intrinsically faint events are best accounted for by including a realistic distribution of the absolute peak magnitudes when deriving the CCSN rate results. For example, the effects of including a large $(30 \%)$ fraction of faint $(M>-15)$ CCSNe was studied by Melinder et al. (2012). As a result, they found an increase of $\sim 30 \%$ in their CCSN rates at $z \sim 0.4$ and $z \sim 0.7$.

\subsection{Missing SNe in Normal Galaxies}

We can now proceed to estimate the missing fraction of $\mathrm{SNe}$ due to high dust extinctions in normal galaxies $\left(L_{\mathrm{IR}}<\right.$ $\left.10^{11} L_{\odot}\right)$ using all the available information we have gathered. A correction for the dust extinction along the lines of Riello \& Patat (2005) can be used to compensate for the CCSNe missed due to inclination effects. However, this should be combined

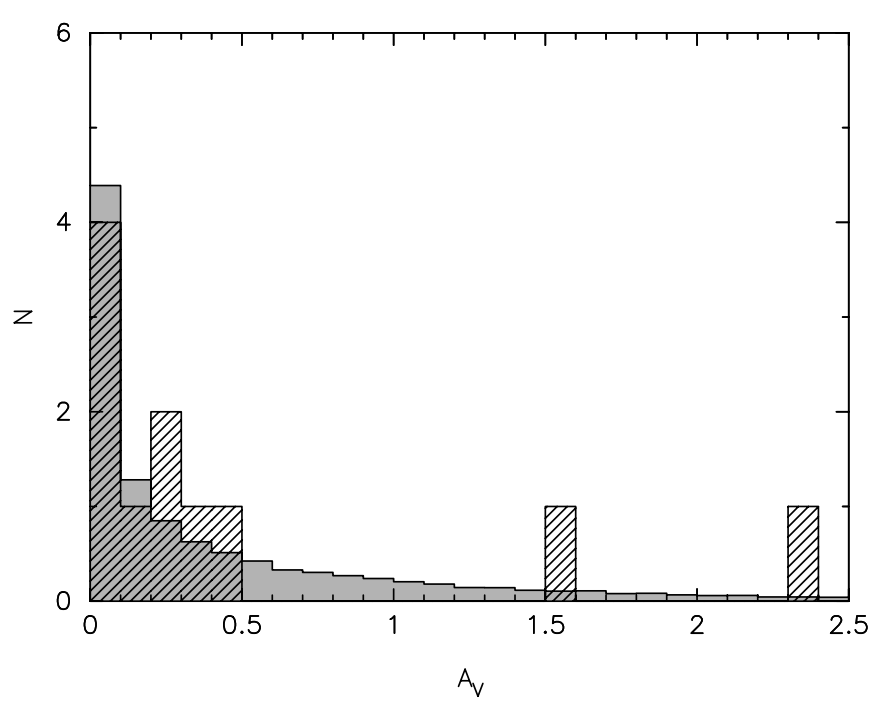

Figure 1. Comparison between the observed (hatched area) and the predicted (gray area) distribution of extinctions $\left(A_{\mathrm{V}}\right)$ for CCSN host galaxy inclinations between $0^{\circ}$ and $60^{\circ}$. The CCSNe closer than $12 \mathrm{Mpc}$ have been included and the two outliers (SNe 2002hh and 2009hd) have been excluded. The two events with the highest extinctions in the sample are SNe 2008ax and 2005at. The predicted distribution (with the absolute level scaled to match the number of observed SNe) follows that of Riello \& Patat (2005) for $B / T=0.0$ but assuming $\tau_{V}(0)=2.5$

with a fraction of $\mathrm{SNe}$ with significantly higher extinctions than predicted by these models when considering a realistic distribution of extinctions for CCSNe in normal galaxies. We concentrate on the sample of $13 \mathrm{CCSNe}$ with host galaxy inclinations of $0^{\circ}-60^{\circ}$ (see Table 3 ). The host galaxies of these events have IR luminosities ranging between $\sim 10^{9}$ and $\sim 3 \times$ $10^{10} L_{\odot}($ see Column 12, Table 1$)$. The observed average extinction calculated for the $0^{\circ}-60^{\circ}$ bin of $\left\langle A_{\mathrm{V}}\right\rangle=1.00$ (sample 1 in Table 3 ) is significantly higher than the predicted value, $\left\langle A_{\mathrm{V}}\right\rangle=0.48$ (although the median extinctions are similar). However, if excluding the two events with the highest host galaxy extinctions (SNe 2002hh and 2009hd with $A_{\mathrm{V}}=3.7$ and 4.1, respectively), the observed average extinction becomes $\left\langle A_{\mathrm{V}}\right\rangle=$ 0.48 (sample 2 in Table 3 ), which is identical to the predicted value (the median value does not change). The standard deviations of the observed and predicted distributions are also now very similar $(0.74$ versus 0.68$)$.

In Figure 1, the observed and predicted extinction distributions are compared after excluding the two outliers with $A_{\mathrm{V}} \gtrsim$ 3.7. We note that only about $0.3 \%$ of the events in the predicted distribution suffer from extinctions higher than $A_{\mathrm{V}}=$ 3.7. This indicates that the simple model with a homogeneous dust distribution used for the simulations does not produce a realistic distribution of line-of-sight extinctions for $\mathrm{SNe}$ in spiral galaxies. CCSNe are observed to be associated with star-forming regions in their host galaxies (e.g., Anderson et al. 2012) and therefore higher extinction values local to the $\mathrm{SNe}$ can be expected. However, we also note that the number of $\mathrm{SNe}$ in our current sample is rather small, making any detailed comparison less reliable.

The two outlier events with host galaxy extinctions of $A_{\mathrm{V}}=$ 3.7-4.1 (or $A_{\mathrm{B}}=4.9-5.5$ ) would be the ones most likely missed by optical SN searches in more distant galaxies. For example, a typical Type II-P event with absolute peak magnitude $M(B)=-17.0$ at a redshift of 0.5 would have an apparent peak magnitude of $m(R) \sim 30$ if the host galaxy extinction is $A_{\mathrm{B}}=5$. This is several magnitudes below the limits of the current 


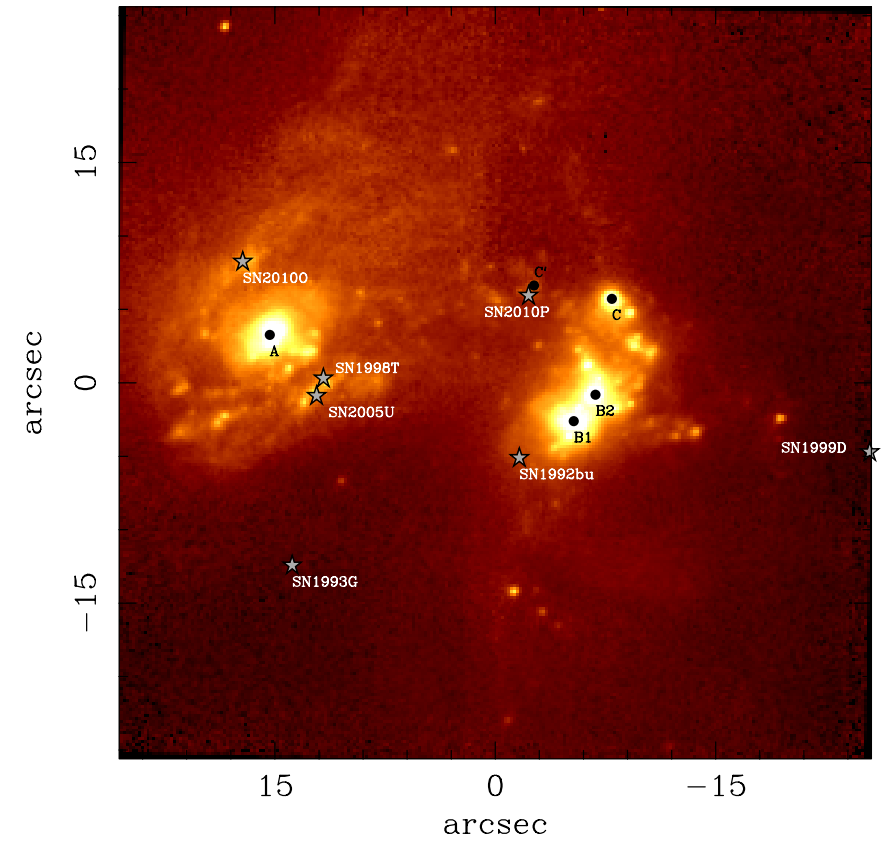

Figure 2. HST/NICMOS F164N image (from Alonso-Herrero et al. 2000) of Arp 299 shown with a square-root scaling to emphasize the extent of the diffuse emission in the circumnuclear regions. This image traces the CCSN activity via the $[\mathrm{Fe} \mathrm{II}] 1.644 \mu \mathrm{m}$ line. The positions of the main nuclei A, B1 and B2, sources $\mathrm{C}$ and $\mathrm{C}^{\prime}$, and the SNe discovered at optical or IR wavelengths are indicated.

(A color version of this figure is available in the online journal.)

high- $z$ SN searches (e.g., Dahlen et al. 2012; Melinder et al. 2012). At higher redshifts the effects of the extinction would be even more severe for an optical SN search since shorter rest-frame wavelengths are observed. However, we note that $\mathrm{SN}$ searches are often optimized for detecting SNe at rest-frame $B$ and $V$ bands, since the SN spectral energy distribution (SED) peaks in this range. For example, searches aiming at a redshift around 0.5 (e.g., Bazin et al. 2009; Melinder et al. 2011) have been typically observing in $R$ and $I$ bands and searches reaching for $z \sim 1$ (e.g., Dahlen et al. 2012) in the $z$-band. The ongoing SN search program (e.g., Rodney et al. 2012) as a part of the CANDELS project (e.g., Grogin et al. 2011) is aiming to detect SNe at $z \sim 1.5-2$ and observes in the near-IR $J$ and $H$ bands. Therefore, we now use the sample of 13 CCSNe within the $0^{\circ}-60^{\circ}$ bin and the two outlier events with the highest host galaxy extinctions to estimate the fraction of CCSNe likely missed by rest-frame optical surveys in normal galaxies to be $15_{-10}^{+21} \%$.

\section{SUPERNOVA BUDGET OF LIRGS AND ULIRGS}

We next consider the fraction of missing $\mathrm{SNe}$ in galaxies with $L_{\mathrm{IR}}>10^{11} L_{\odot}$, i.e., LIRGs and ULIRGs. The interacting system Arp 299 (=IC $694+$ NGC 3690) is one of the most nearby examples of an LIRG at a luminosity distance of $46.7 \mathrm{Mpc}$. Its IR luminosity $L_{\mathrm{IR}}=\mathrm{L}[8-1000 \mu \mathrm{m}]$ of $7.3 \times 10^{11} L_{\odot}$ (adopted from Sanders et al. 2003 and scaled to the assumed distance) indicates a very high CCSN rate of $\sim 2 \mathrm{yr}^{-1}$ which is one of the highest expected in local galaxies. Arp 299 has been the target of several SN searches and over the last two decades a total of seven SNe have been discovered at optical or near-IR wavelengths within its circumnuclear regions, $\sim 1-4 \mathrm{kpc}$ from the main galaxy nuclei A and B1 (see Figure 2). It is therefore well suited for investigating the fraction of $\mathrm{SNe}$ missed by optical observations in one of the best-observed local LIRGs.
Table 4

SN Searches in Arp 299

\begin{tabular}{lccc}
\hline \hline Telescope & Band & Period & SNe \\
\hline Leuschner $^{\mathrm{a}}$ & vis & Before 1998 & 1993G \\
Richmond & VIS & 1988 Dec-1991 Jun & $\ldots$ \\
BAOSS $^{\mathrm{b}}$ & VIS & & SN 1998T \\
LOSS $^{\mathrm{c}}$ & VIS & 1998-ongoing & 1999D (1998T, 2005U) \\
\hline IRTF & NIR & 1992 Mar-1993 Dec & $1992 \mathrm{bu}$ \\
WIRO & NIR & 1993 Feb-1993 Dec & $\ldots$ \\
TNG & NIR & 1999 Oct-2001 Oct & $\ldots$ \\
USNO & NIR & 2001 Feb-2004 May & $\ldots$ \\
WHT & NIR & 2002 Jan-2005 Jan & 2005U \\
NOT & NIR & 2005 Mar-ongoing & 2010P (2010O) \\
Gemini-N & NIR & 2008 Apr-ongoing & $(20100,2010 P)$ \\
\hline
\end{tabular}

Notes.

a Leuschner Observatory Supernova Search.

b Beijing Astronomical Observatory Supernova Survey.

${ }^{c}$ Lick Observatory Supernova Search.

In Section 4, we make use of these estimates to also extrapolate the results to higher redshifts.

\subsection{Observed SNe in Arp 299}

During the last two decades, Arp 299 has been the target of a number of professional and amateur SN searches (see Table 4 for summary). At optical wavelengths, Arp 299 has been the target of at least four professional SN searches. In the early 1990s it was monitored by the Leuschner Observatory SN Search that was the predecessor of the Lick Observatory SN search (LOSS), the Beijing Observatory SN search, the Richmond et al. (1998) search, and finally the LOSS. Arp 299 was also included in the "optimal" galaxy sample of the LOSS with their SN search data between 1998 March and 2008 December used for the SN rate calculation (Leaman et al. 2011; Li et al. 2011a, 2011b). SN 1993G was discovered by the Leuschner Observatory SN Search, SN $1998 \mathrm{~T}$ by the Beijing Observatory SN search, and SN 1999D by the LOSS. LOSS also detected SNe 1998T and 2005U, although they were originally discovered elsewhere. The observed $B-V$ color of SN $1993 \mathrm{G}$ indicated that the SN was virtually unreddened (Tsvetkov 1994). SN 1998 T was spectroscopically classified by Li et al. (1998) reporting strong P-Cygni profiles of $\mathrm{He} \mathrm{I}$ lines between 510 and $900 \mathrm{~nm}$. Therefore, the extinction toward SN 1998T was also likely low. In the case of SN 1999D the typing spectrum featured a very blue continuum (Jha et al. 1999), strongly suggesting a low extinction. For these three events we therefore assume that the host galaxy extinction was close to zero.

Van Buren et al. (1994) conducted a $K$-band survey for SNe in starburst galaxies at the NASA $3.0 \mathrm{~m}$ Infrared Telescope Facility (IRTF). As a part of their search Arp 299 was observed in at least four epochs between 1992 March and 1993 December, yielding the discovery of SN 1992 bu in their $K$-band images. The SN is located at a projected distance of $\sim 1.0 \mathrm{kpc}$ from the nucleus B1 of the galaxy. SN 1992bu has no spectroscopic classification and, based on just the $K$-band photometry, estimating the extinction is difficult. However, as a part of their analysis, Anderson et al. (2011) noted that SN 1992bu falls on a bright star-forming region and has a small galactocentric distance. Hence, they find it consistent with being a stripped envelope core-collapse event.

A more recent near-IR SN search targeting nearby starburst galaxies was carried out in the $K^{\prime}$ band by Grossan et al. (1999). 
The observations were performed with the $2.3 \mathrm{~m}$ telescope at the Wyoming IR Observatory (WIRO). As part of the search, five observations of Arp 299 were taken between 1993 February and December. Arp 299 was also targeted for a near-IR $K$-band SN search carried out with the $3.6 \mathrm{~m}$ Telescopio Nazionale Galileo (TNG) between 1999 October and 2001 October including nine observations (Mannucci et al. 2003). Another near-IR $K$-band SN search observing Arp 299 was carried out using the $1.55 \mathrm{~m}$ U.S. Naval Observatory telescope at Flagstaff (e.g., Dudley et al. 2008). During the search, Arp 299 was observed in a total of 19 epochs between 2001 February and 2004 May (C. C. Dudley 2012, private communication). However, no confirmed SNe were reported in Arp 299 as a result of these three searches.

Arp 299 was also monitored in a near-IR $K$-band for a total of eight epochs between 2002 January and 2005 January as a part of the nuclear SN search campaign with the William Herschel Telescope resulting in the discovery of SN 2005U (Mattila et al. 2004, 2005a). The near-IR color of SN 2005U indicated only a modest extinction. The SN was spectroscopically classified as a Type-II event, probably within a few weeks of the explosion (Modjaz et al. 2005). A further spectrum of SN 2005U was obtained by Leonard \& Cenko (2005) showing it to resemble the spectrum of the Type IIb SN 1993J over a month past its explosion with no evidence of substantial host galaxy extinction (D. C. Leonard 2011, private communication; M. Modjaz et al., in preparation).

More recently, we have been monitoring Arp 299 for SNe in the $K$ band using the $2.6 \mathrm{~m}$ Nordic Optical Telescope (NOT) every few months for a total of $\sim 20$ epochs, resulting in the discovery of SN 2010P (Mattila \& Kankare 2010) and the detection of SN 2010O, which was also discovered at optical wavelengths (Newton et al. 2010). SN 20100 was spectroscopically classified as a Type $\mathrm{Ib} / \mathrm{c}$ event around maximum light and a host galaxy extinction of $A_{\mathrm{V}} \sim 1.9$ was estimated from prominent $\mathrm{Na}$ I D absorption lines from the host galaxy (Mattila et al. 2010). Between 2008 and 2011 we have also been monitoring Arp 299 using high spatial resolution observations with laser guide star adaptive optics on the Gemini North telescope as part of a sample of nearby LIRGs (see Kankare et al. 2008, 2012). Our most recent $K$-band observations from the NOT and the Gemini-N telescope were obtained in 2012 March and 2012 February, respectively.

Recently, Anderson et al. (2011) analyzed the circumnuclear SN population in Arp 299 finding a relatively high fraction of stripped envelope events (Types $\mathrm{Ib}$ and $\mathrm{IIb}$ ) relative to other Type II SNe. They suggested that this excess could be explained by the young age of circumnuclear star formation in Arp 299 such that we would now be witnessing the explosions of the most massive stars formed. Alternatively, the excess of stripped envelope events might be explained by a top-heavy initial mass function (IMF) favoring the formation of the most massive stars.

\subsubsection{The Highly Obscured SN $2010 P$}

SN 2010P was discovered via image-subtraction techniques in near-IR images obtained using NOTCam on January 18.2 and 23.1 UT. The approximate magnitudes of SN 2010P on 2010 January 23.1 were $m_{I}=18.3, m_{J}=16.8, m_{H}=16.2$, and $m_{K}=15.9$ (Mattila \& Kankare 2010). Nothing is visible at this position on NOTCam $K$-band images taken on 2009 November 27.2 (limiting mag 17.5). We note that SN 2010P was also detectable by image subtraction in our I-band image obtained using StanCam on January 23.1 on the NOT. However, the $\mathrm{SN}$ was not detected in our $R$-band images from the
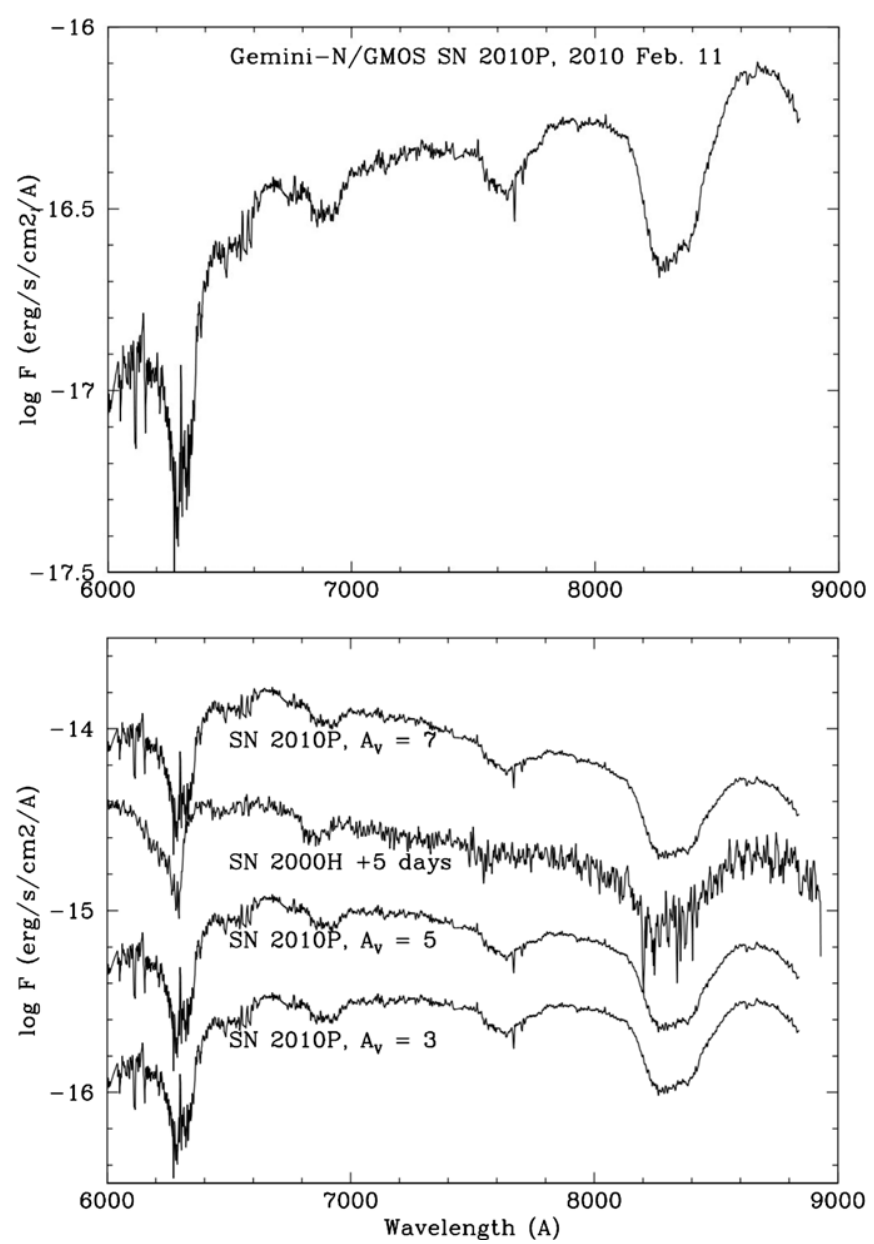

Figure 3. Gemini-N/GMOS spectrum of SN 2010P within the nuclear regions of Arp 299. Top: the observed spectrum before dereddening. Bottom: the spectrum after dereddening by $A_{\mathrm{V}}=3,5$, and 7 compared with the Type IIb/Ib SN $2000 \mathrm{H}$ (corrected for the galactic reddening according to Schlegel et al. (1998)).

same instrument. A radio follow-up using MERLIN between 2010 January 29 and February 1 at $4.99 \mathrm{GHz}$ resulted in a non-detection for SN 2010P (Beswick et al. 2010). The absolute magnitude and colors of 2010P are consistent with the CCSN template light curves from Mattila \& Meikle (2001) with a likely extinction of $A_{\mathrm{V}} \sim 5$.

In order to allow spectroscopic typing and a more reliable extinction determination, we obtained long-slit spectroscopy of SN 2010P with the Gemini Multi-Object Spectrograph (GMOS; Hook et al. 2004) attached to the Gemini-N Telescope as a part of the programGN-2010A-Q-40 (PI: S. Ryder). Four exposures of $900 \mathrm{~s}$ each were taken on the night of 2010 February $11 \mathrm{UT}$ using the R400 grating and a 0.'5 wide slit to deliver a resolution of $R \sim 1900$. The data were reduced and combined using V1.10 of the gemini package within IRAF. ${ }^{9}$

The spectrum of SN 2010P has a red continuum and very little signal at wavelengths shorter than $6000 \AA$ (see Figure 3), indicative of a high extinction. Despite significant reddening, the spectrum was found to be consistent with an H-deficient CCSN. Comparison with a library of SN spectra using both the "GELATO" code (Harutyunyan et al. 2008) and

\footnotetext{
9 IRAF is distributed by the National Optical Astronomy Observatory, which is operated by the Association of Universities for Research in Astronomy, Inc. under cooperative agreement with the National Science Foundation.
} 
Table 5

The Optical/Near-IR SNe of Arp 299

\begin{tabular}{lccccc}
\hline \hline SN & SN Type & $\begin{array}{c}\text { Distance } \\
(\mathrm{kpc})\end{array}$ & $A_{\mathrm{V}}$ & Band & Discovery Date \\
\hline $1992 \mathrm{bu}$ & $\ldots$ & $1.0(\mathrm{~B} 1)$ & $\ldots$ & NIR & 1992 Mar 9 \\
$1993 \mathrm{G}$ & IIL & $3.6(\mathrm{~A})$ & $\sim 0$ & VIS & 1993 Mar 5 \\
$1998 \mathrm{~T}$ & II & $1.1(\mathrm{~A})$ & $\sim 0$ & VIS & 1998 Mar 3 \\
$1999 \mathrm{D}$ & $\mathrm{Ib}$ & $4.5(\mathrm{~B} 1)$ & $\sim 0$ & VIS & 1999 Jan 17 \\
$2005 \mathrm{U}$ & $\mathrm{IIb}$ & $1.2(\mathrm{~A})$ & $\sim 0$ & NIR & 2005 Jan 30 \\
$2010 \mathrm{O}$ & $\mathrm{Ib}$ & $1.2(\mathrm{~A})$ & $\sim 2$ & VIS & 2010 Jan 24 \\
$2010 \mathrm{P}$ & $\mathrm{Ib} / \mathrm{IIb}$ & $0.2\left(\mathrm{C}^{\prime}\right)$ & $\sim 5$ & NIR & 2010 Jan 18 \\
\hline
\end{tabular}

Notes. The offsets are from the nearest nucleus (given in brackets) using their coordinates at $8.46 \mathrm{GHz}$ from Romero-Cañizales et al. (2011). For identifications of the supernovae and the different nuclei see Figure 2.

the "SuperNova IDentification" code (Blondin \& Tonry 2007) yielded good matches to several Type Ib and Type IIb SNe between one and three weeks after maximum light (the spectrum of SN 2010P was dereddened by 5 mag of extinction in $A_{\mathrm{V}}$ before using the "Gelato" code). In Figure 3, we illustrate this by comparing the spectrum of SN 2010P, dereddened by $A_{\mathrm{V}}=3,5$, and 7 , with that of the Type $\mathrm{IIb} / \mathrm{Ib}$ event SN $2000 \mathrm{H}$ at 5 days past maximum from Branch et al. (2002). For this the reddening law of Cardelli et al. (1989) was assumed with a value of $R_{\mathrm{V}}=3.1$. Following Elmhamdi et al. (2006) we assume the host galaxy extinction toward SN $2000 \mathrm{H}$ to be small. A very high amount of extinction toward SN 2010P is also consistent with its near-IR light curves, colors, and absolute magnitude.

SN 2010P has a projected distance of only $\sim 200 \mathrm{pc}$ from the source $C^{\prime}$, but $\sim 1.3 \mathrm{kpc}$ from the source $\mathrm{C}$ (see Figure 2). Although the region $\mathrm{C}^{\prime}$ is a strong radio source, it is much less prominent at IR wavelengths with only $\sim 10 \%$ of source $\mathrm{C}$ at $2.2 \mu \mathrm{m}$ increasing to $\sim 1 / 3$ at $18 \mu \mathrm{m}$ (Charmandaris et al. 2002). Based on the optical depth of the $9.7 \mu \mathrm{m}$ absorption feature, Alonso-Herrero et al. (2009) estimated an extinction of $A_{\mathrm{V}}=12$ toward source $\mathrm{C}^{\prime}$, which is similar to their estimated extinction for nucleus B1. Having only $\sim 200 \mathrm{pc}$ projected distance from the source $C^{\prime}$ and a substantial host galaxy extinction, we consider SN 2010P as a genuine nuclear SN in the following analysis. Other SNe with high host galaxy extinctions previously detected in LIRGs include SNe 2001db $\left(A_{\mathrm{V}} \sim 5.5\right.$; Maiolino et al. 2002), 2004ip ( $A_{\mathrm{V}} \geqslant 5$; Mattila et al. 2007), 2008cs $\left(A_{\mathrm{V}} \sim\right.$ 16; Kankare et al. 2008), and 2011hi $\left(A_{\mathrm{V}}=5-7\right.$; Kankare et al. 2012; Romero-Cañizales et al. 2012), all discovered in the nearIR $K$ band similar to SN 2010P.

\subsection{Optical/near-IR CCSN Rate of Arp 299}

Including only the SNe discovered from 1998 onward when the LOSS and a number of near-IR SN searches monitoring Arp 299 were active, we have a total of five SNe discovered during a period of $\sim 14 \mathrm{yr}$ (1998 March-2012 March). The projected distances of these SNe from the nearest nuclei are listed in Table 5. All of these except for SN 2010P range between $\sim 1$ and $\sim 5 \mathrm{kpc}$.

Despite the numerous efforts at optical and near-IR wavelengths to detect $\mathrm{SNe}$ within the innermost nuclear regions of Arp 299 during the last two decades, only one (SN 2010P) has been detected close to one of the nuclei of Arp 299. This is consistent with the high dust extinctions therein, which make the $\mathrm{SNe}$ too dim to be detectable. In order to estimate the actual fraction of SNe missed in Arp 299 due to dust extinction, we need to estimate the fraction of star formation and thus CCSNe in the nuclear and circumnuclear regions.

From the observations we know that at least five SNe occurred in Arp 299 during the $14 \mathrm{yr}$ period. Using these five SNe we can now estimate a lower limit for the optical/near-IR CCSN rate in Arp 299 over the period of $14 \mathrm{yr}$ of $0.36_{-0.15}^{+0.24} \mathrm{yr}^{-1}$. Of the five SNe all except SN 2010P were detected by the optical SN searches (although SN 2005U was originally discovered in the near-IR) and had a relatively low extinction. SN 2010P was marginally detected in our I-band images from the NOT (after its initial discovery in the near-IR) and was not detectable in $R$-band images from the same telescope. It had a high extinction of $A_{\mathrm{V}} \sim 5$ and therefore most likely would not be detected in the current optical SN searches. In fact, shortly before our discovery report on SN 2010P, the discovery of SN 20100 was reported by Newton et al. (2010) in the course of the Puckett Observatory Supernova Search. They did not detect SN 2010P in their unfiltered CCD images. Therefore, we assume that 4 out of the $5 \mathrm{SNe}$ discovered in Arp 299 during the $14 \mathrm{yr}$ period were optically detectable. This yields a lower limit for the rate of optically detectable circumnuclear SNe in Arp 299 of $0.29_{-0.14}^{+0.23} \mathrm{yr}^{-1}$

\subsection{Predicted CCSN Rate of Arp 299}

After having determined the observed SN rate in Arp 299, we now turn to estimating the predicted rate based on the SFR in order to calculate the fraction of SNe hidden by dust. The CCSN rate within the highly obscured innermost nuclear regions of Arp 299 can be estimated either via infrared or radio luminosity. Radio luminosity based estimates for the nuclear SN rate of Arp 299 from VLA measurements have been presented by Neff et al. (2004) who estimated (scaled to the distance of $46.7 \mathrm{Mpc}$ assumed here) $0.65-1.3 \mathrm{yr}^{-1}, 0.13-0.26 \mathrm{yr}^{-1}$, and $0.07-0.13$ and $0.04-0.08 \mathrm{yr}^{-1}$ for the nuclei $\mathrm{A}$ and $\mathrm{B} 1$, and sources $\mathrm{C}$ and $C^{\prime}$, respectively (see Figure 2 for the identifications of the different nuclei). Alonso-Herrero et al. (2000) estimated $0.8 \mathrm{yr}^{-1}$ and $0.15 \mathrm{yr}^{-1}$ for nuclei $\mathrm{A}$ and $\mathrm{B}$, respectively (again scaled to $46.7 \mathrm{Mpc}$ ). More, recently, Romero-Cañizales et al. (2011) estimated a lower limit of $0.28_{-0.15}^{+0.27} \mathrm{yr}^{-1}$ for the CCSN rate of nucleus B1 based on archival VLA observations over a period of $11 \mathrm{yr}$, which we will adopt as the more direct estimate to use in this study. Pérez-Torres et al. (2009) and Bondi et al. (2012) made use of extremely high spatial resolution European VLBI network $(\mathrm{EVN})$ radio observations of the innermost nuclear regions of Arp 299-A. They find clear evidence for at least two new radio supernovae (RSNe) in their observations separated by two years therefore implying a lower limit for the CCSN rate in nucleus $\mathrm{A}$ of $\sim 0.8 \mathrm{yr}^{-1}$.

We can also estimate the CCSN rates indirectly from the galaxy IR luminosity. Charmandaris et al. (2002) estimated that IC 694 (nucleus A), NGC 3690 (nucleus B1+B2), and sources $\mathrm{C}+\mathrm{C}^{\prime}$ emit approximately $39 \%, 20 \%$, and $10 \%$ of the total IR luminosity of Arp 299, respectively, with the remaining $\sim 31 \%$ originating from the circumnuclear regions (see Column 2, Table 7). The rather large contribution of the circumnuclear regions to the total luminosity is also supported by the $\mathrm{H} \alpha$ observations by García-Marín et al. (2006) showing significant amounts of star formation in the spiral arms of IC 694 and also to the west of nucleus B1 in NGC 3690. Adopting the galaxy IR luminosity of $7.3 \times 10^{11} L_{\odot}$ and an empirical relation between the IR luminosity and CCSN rate $\left(r_{\mathrm{SN}}=2.7 \times 10^{-12} \times L_{\mathrm{IR}} / L_{\odot}\right.$ $\mathrm{yr}^{-1}$ ) from Mattila \& Meikle (2001), we have a total CCSN rate of $\sim 2.0 \mathrm{yr}^{-1}$ for the entire system (see also the discussion in 
Romero-Cañizales et al. 2011). However, this IR-luminositybased CCSN rate estimate could have a significant uncertainty because the empirical relation is based on only three nearby starburst galaxies NGC 253, M 82, and NGC 4038/9 with IR luminosities substantially lower than of Arp 299.

\subsection{Modeling the SEDs of Nuclei $A$ and $B 1+B 2$}

As a more accurate approach we estimate the CCSN rates for the Arp 299 nuclei by modeling their SEDs. This approach has the advantage that it can take into account the effects of the starburst age as well as the possible contribution of an active galactic nucleus (AGN) to the IR luminosities of the nuclei. For this purpose we have used low-resolution mid-IR Spitzer/IRS (SL+LL setting; 5-38 $\mu \mathrm{m}$ range) spectra covering $\sim 10^{\prime \prime} .4 \times$ $10^{\prime \prime} 4$ rectangular regions (see the lower panel of Figure 5 in Alonso-Herrero et al. 2009) centered on the nuclei A and B1+B2. These spectra were obtained on 2004 April 15 and have already been reported in Alonso-Herrero et al. (2009). In addition, we included IRAS 12, 25, 60, and $100 \mu \mathrm{m}$ fluxes from Sanders et al. (2003) assigned to nuclei A and B1+B2 according to the estimated contributions from Charmandaris et al. (2002). In order to obtain a reasonable match between the Spitzer spectra and the IRAS 12 and $25 \mu$ m fluxes, we multiplied both the Spitzer/IRS spectra by 1.3. This value is well within the uncertainties expected in the estimates of Charmandaris et al. (2002) for the fractions of IR luminosities arising from the different nuclei of Arp 299 and the calibration errors of the IRAS and Spitzer/IRS data.

For modeling the SED of Arp 299 we use a grid of AGN torus models that have been computed with the method of Efstathiou \& Rowan-Robinson (1995) and a grid of starburst models that have been computed with the method of Efstathiou et al. (2000). For the AGN torus models we use the tapered disk models computed with the method of Efstathiou \& Rowan-Robinson (1995) and described in more detail in A. Efstathiou et al. (2012, in preparation). These models considered a distribution of grain species and sizes, multiple scattering and a density distribution that followed $r^{-1}$ where $r$ is the distance from the central source. The models assumed a smooth distribution of dust, so they are a good approximation of the density distribution in the torus if the mean distance between clouds is small compared with the size of the torus, but they have been quite successful in fitting the SEDs of AGNs even in cases where mid-infrared spectroscopy is available (e.g., A. Efstathiou et al. 2012, in preparation). In this grid of models we consider four discrete values for the equatorial $1000 \AA$ optical depth $(500,750,1000,1250)$, three values for the ratio of outer-to-inner disk radii $(20,60,100)$ and three values for the opening angle of the disk $\left(30^{\circ}, 45^{\circ}\right.$, and $60^{\circ}$ ). The spectra are computed for inclinations which are equally spaced in the range $0-\pi / 2$.

Efstathiou et al. (2000) presented a starburst model that combined the stellar population synthesis model of Bruzual \& Charlot (2003), a detailed radiative transfer that included the effect of small grains and polycyclic aromatic hydrocarbons (PAHs), and a simple evolutionary scheme for the molecular clouds that constitute the starburst. The model predicts the SEDs of starburst galaxies from the ultraviolet to the millimeter as a function of the age of the starburst and the initial optical depth of the molecular clouds. In this paper, we use a sequence of models that have been computed with an updated dust model (Efstathiou \& Siebenmorgen 2009). We assume an exponentially declining SFR with an $e$-folding time of $20 \mathrm{Myr}$. The choice of $20 \mathrm{Myr}$ is supported by fits to the far-IR color-color diagrams (e.g.,
Table 6

The Starburst Parameters Obtained from the SED Fits for Arp 299

\begin{tabular}{lcrccc}
\hline \hline Region & Age & \multicolumn{1}{c}{$\tau_{\mathrm{V}}$} & $\begin{array}{c}L_{\mathrm{IR}} \\
\left(L_{\odot}\right)\end{array}$ & $\langle$ SFR $\rangle$ & SNR \\
\hline A & $45 \mathrm{Myr}$ & 75 & $2.45 \times 10^{11}$ & $90 M_{\odot} \mathrm{yr}^{-1}$ & $0.76 \mathrm{yr}^{-1}$ \\
$\mathrm{~B} 1+\mathrm{B} 2$ & $55 \mathrm{Myr}$ & 100 & $1.19 \times 10^{11}$ & $56 M_{\odot} \mathrm{yr}^{-1}$ & $0.33 \mathrm{yr}^{-1}$ \\
\hline
\end{tabular}

Notes. The IR luminosities of the starburst component are listed in Column 4. The total IR luminosity of nucleus $\mathrm{B}$ is $1.49 \times 10^{11} L_{\odot}$ including a $20 \%$ contribution from an AGN. An exponentially declining SFR with an $e$-folding time of 20 Myr has been assumed. The SFRs given in Column 5 have been averaged over the duration of the starburst. The corresponding CCSN rates (SNRs) are given in Column 6.

Efstathiou et al. 2000), to the SEDs of ULIRGs (e.g., Farrah et al. 2003), and to the Spoon diagram (Rowan-Robinson \& Efstathiou 2009). Our model fits to the Spitzer/IRS (SL+LL) spectra are shown in Figure 4. For nucleus A, no AGN component is required, whereas for nucleus $\mathrm{B} 1+\mathrm{B} 2$ we find a $20 \%$ contribution by an AGN to the total IR $(8-1000 \mu \mathrm{m})$ luminosity. This agrees well with the recent findings of AlonsoHerrero et al. (2012). The best-fit dusty torus parameters we find for the AGN are $\tau(1000 \AA)=500, r_{\text {outer }} / r_{\text {inner }}=20$, an opening angle of $60^{\circ}$, and an inclination of $45^{\circ}$. The resulting starburst parameters are listed in Table 6.

The CCSN rates were then estimated in the following way. The stellar population synthesis model of Bruzual \& Charlot (2003) makes a prediction of the CCSN rate $\operatorname{SNR}(t)$ at a time $t$ after star formation in an instantaneous burst. The starburst model of Efstathiou et al. (2000) predicts the spectrum of this instantaneous burst at time $t$ and assumes a star formation history for the starburst. It is therefore possible to calculate selfconsistently the CCSN rate at different stages in the evolution of a starburst by convolving the star formation history with $\operatorname{SNR}(t)$. This results in CCSN rates of 0.76 and $0.33 \mathrm{yr}^{-1}$ for nuclei $\mathrm{A}$ and $\mathrm{B} 1+\mathrm{B} 2$, respectively.

\subsection{The Missing SNe in Arp 299}

The IR luminosities for the different components of Arp 299 are listed in Table 7 (Column 2) adopting their fractional contributions from Charmandaris et al. (2002) and a total IR luminosity for the system of $7.3 \times 10^{11} L_{\odot}$. We compared our SED fit based model IR luminosities (see Table 6) of nuclei $\mathrm{A}$ and $\mathrm{B} 1+\mathrm{B} 2$ with the "observed" ones obtained as described above. The model luminosity for nucleus A of $2.45 \times 10^{11} L_{\odot}$ is slightly lower than the observed $L_{\mathrm{IR}}=2.85 \times 10^{11} L_{\odot}$. However, we note that the simple gray body models of Charmandaris et al. (2002) also gave lower IR luminosities for the Arp 299 nuclei than the observed values. The total (AGN+starburst) model IR luminosity for nucleus $\mathrm{B} 1+\mathrm{B} 2$ of $1.49 \times 10^{11} L_{\odot}$ is almost identical to the observed value of $1.46 \times 10^{11} L_{\odot}$.

For the sources $\mathrm{C}+\mathrm{C}^{\prime}$ we estimate the $\mathrm{CCSN}$ rates from the observed IR luminosity using the empirical relation of Mattila $\&$ Meikle (2001). This yields a CCSN rate of $0.20 \mathrm{yr}^{-1}$ which is also consistent with the radio-based estimate of $0.16 \pm$ $0.05 \mathrm{yr}^{-1}$. We note that the same approach would yield CCSN rate estimates of 0.77 and $0.39 \mathrm{yr}^{-1}$ for nuclei $\mathrm{A}$ and $\mathrm{B} 1+\mathrm{B} 2$ very similar to the IR SED modeling based values (note that a slightly higher value for nucleus $\mathrm{B} 1+\mathrm{B} 2$ is as expected due to the $20 \%$ AGN contribution in B1). For the total nuclear CCSN rate we combine the results from the IR SED modeling of nucleus $\mathrm{A}$ and $\mathrm{B} 1+\mathrm{B} 2$, with the IR luminosity derived rate for sources $\mathrm{C}+\mathrm{C}^{\prime}$ to obtain a predicted nuclear CCSN rate of $1.29 \mathrm{yr}^{-1}$. 

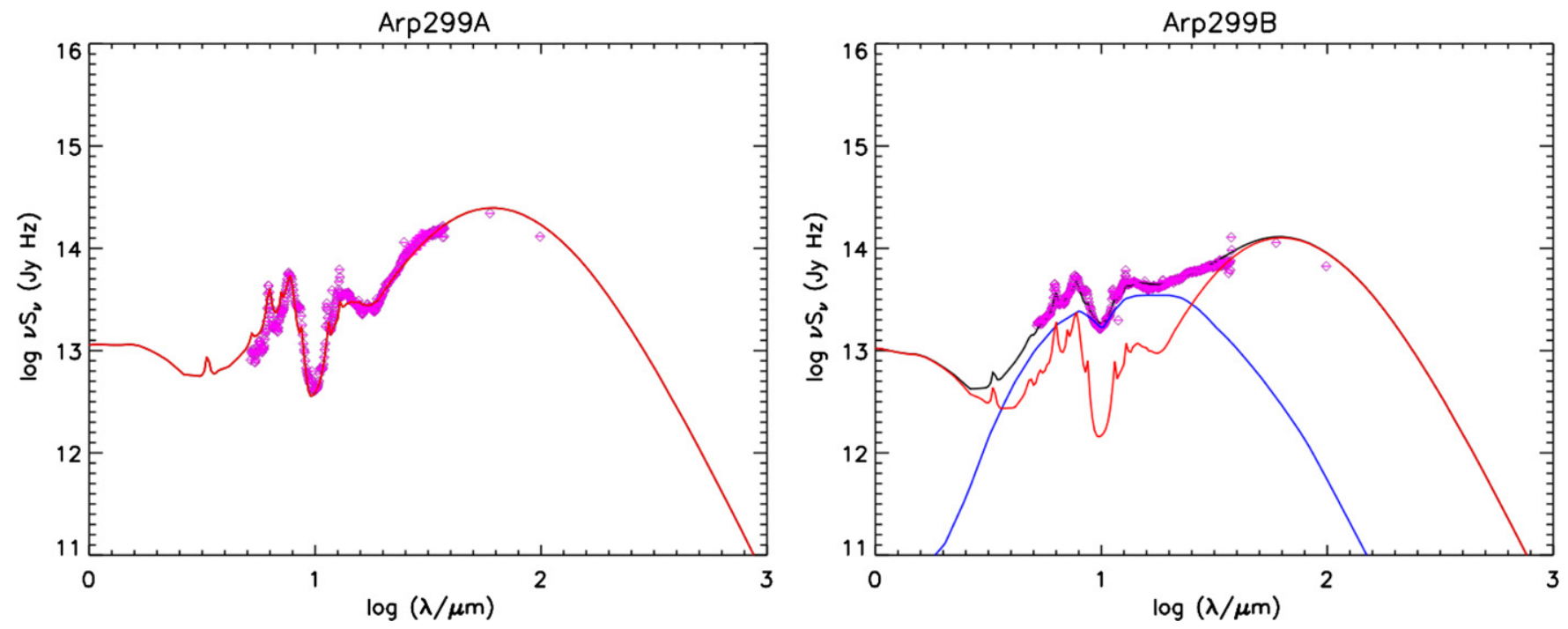

Figure 4. Starburst (red line) and AGN (blue line) model fits to the mid-IR SED of the nuclei A and B1+B2 of Arp 299. For nucleus A, no AGN component is required, whereas for nucleus B1+B2, there is a $20 \%$ contribution by an AGN. The starburst model parameters are given in Table 6.

Table 7

The SN Budget of Arp 299

\begin{tabular}{lccccccc}
\hline \hline Region & $L_{\mathrm{IR}}$ & \multicolumn{3}{c}{ Predicted SNR $\left(\mathrm{yr}^{-1}\right)$} & & \multicolumn{2}{c}{ Observed SNR $\left(\mathrm{yr}^{-1}\right)$} \\
\cline { 3 - 5 } & $\left(\times 10^{11} L_{\odot}\right)$ & $\mathrm{IR}$ & Radio & $H_{\alpha}$ & & Optical+NIR & Optical \\
\hline $\mathrm{A}$ & 2.85 & 0.76 & $>0.8$ & $\ldots$ & $\ldots$ & $\ldots$ \\
$\mathrm{B} 1+\mathrm{B} 2$ & 1.46 & 0.33 & $>0.28_{-0.15}^{+0.27}$ & $\ldots$ & $\ldots$ & $\ldots$ \\
$\mathrm{C}+\mathrm{C}^{\prime}$ & 0.73 & 0.20 & $\sim 0.16 \pm 0.05$ & $\ldots$ & & $>0.07_{-0.06}^{+0.17}$ & $\ldots$ \\
Circumnuclear & 2.26 & 0.61 & $\ldots$ & 0.30 & & $>29_{-0.14}^{+0.22}$ & $>0.29_{-0.14}^{+0.22}$ \\
\hline Total & 7.3 & 1.90 & $>1.2$ & 0.30 & & $>0.36$ & $>0.29$ \\
\hline
\end{tabular}

Notes. The predicted IR-based CCSN rates for nuclei A and B1+B2 were obtained by radiative transfer modeling of their IR SEDs, whereas the rates for sources $C+C^{\prime}$ and the circumnuclear regions were obtained adopting the fractions of the IR luminosity arising from the different components from Charmandaris et al. (2002) and using the empirical relation between the IR luminosity and CCSN rate from Mattila \& Meikle (2001). These yield a total CCSN rate of $1.90 \mathrm{yr}^{-1}$ for Arp 299. The predicted radio-based CCSN rates for nuclei A and B1+B2, and sources $C+C^{\prime}$ were adopted from Bondi et al. (2012), Romero-Cañizales et al. (2011), and Neff et al. (2004), respectively.

The results from Charmandaris et al. (2002) suggest that $31 \%$ of the total IR luminosity originates outside the nuclei A, $\mathrm{B} 1+\mathrm{B} 2$, and $\mathrm{C}+\mathrm{C}^{\prime}$. Furthermore, Alonso-Herrero et al. (2009) also found evidence from their Spitzer/IRS spectral mapping for significant circumnuclear PAH and [Ne II] emission extending outside the main nuclei in Arp 299. Adopting the empirical relation between the IR luminosity and the CCSN rate, this corresponds to $0.61 \mathrm{yr}^{-1}$ for the circumnuclear regions. We note that the use of the CCSN rate estimate (for unobscured Type II+Ib/c SNe in normal galaxies) in units of the galaxy far-IR luminosity from Cappellaro et al. (1999) would yield a very similar circumnuclear CCSN rate estimate. Furthermore, García-Marín et al. (2006) determined an SFR of $43 M_{\odot} \mathrm{yr}^{-1}$ for the entire Arp 299 system (with the nucleus B1 excluded) based on their estimate for the extinction-corrected $\mathrm{H} \alpha$ luminosity. García-Marín et al. (2006) found that most of the circumnuclear H II regions in Arp 299 only suffer from modest extinctions of typically less than $A_{\mathrm{V}} \sim 1$. Assuming a Salpeter IMF between 0.1 and $125 M_{\odot}$ and CCSN progenitor masses between 8 and $50 M_{\odot}$ (e.g., see the discussion in Melinder et al. 2012 and Dahlen et al. 2012), this corresponds to a CCSN rate of $\sim 0.30 \mathrm{yr}^{-1}$ for Arp 299. Having the $\mathrm{H} \alpha$ emission from Arp 299 arising mostly outside the heavily obscured nuclear regions $\mathrm{A}, \mathrm{B} 1+\mathrm{B} 2$, and $\mathrm{C}+\mathrm{C}^{\prime}$ (in contrast to the IR luminosity), we can consider this as a robust lower limit for the circumnuclear CCSN rate and adopt the IR-luminosity-based value of $0.61 \mathrm{yr}^{-1}$ as an upper limit. Combining these circumnuclear CCSN rate estimates with the nuclear CCSN rate therefore yields a total CCSN rate of 1.59-1.90 $\mathrm{yr}^{-1}$ for Arp 299. In Table 7, our predicted CCSN rates are compared to the observed rates based on radio, optical+NIR, and optical searches. These are broadly consistent with each other and together with the data on the optical SN discoveries in the circumnuclear regions can be used to estimate the missing fraction of SNe in Arp 299.

We are now ready to estimate the missing fractions. Within the nuclear regions, no SNe have been detected by optical observations (and only one, SN 2010P, by near-IR observations) and we therefore assume that $100 \%$ of the $\mathrm{SNe}$ are missed by optical searches. For the circumnuclear regions, we estimated a lower limit for the "optical" CCSN rate of $0.29_{-0.14}^{+0.23} \mathrm{yr}^{-1}$, while the predicted rate is $0.30-0.61 \mathrm{yr}^{-1}$, suggesting a missing fraction of up to $37_{-37}^{+38} \%$ in this region. Compared to the total 
Table 8

The Missing SN Fraction in Different Types of Galaxies

\begin{tabular}{lc}
\hline \hline Galaxy & Missing Fraction \\
\hline Normal (observed) & $15_{-10}^{+21} \%$ \\
Normal (corrected) & $19_{-12}^{+24} \%$ \\
U/LIRG (circumnuclear) & $37_{-37}^{+38} \%$ \\
U/LIRG (nuclear) & $100 \%$ \\
U/LIRG (total) & $83_{-15}^{+9} \%$ \\
\hline
\end{tabular}

Notes. The assumed missing SN fractions in normal galaxies, in the nuclear and circumnuclear regions of U/LIRGs, and their combination (total) used to calculate the missing SN fraction as a function of redshift (see Table 10) according to the assumptions given in Table 9. The missing fractions in normal galaxies are given both as observed and after correcting for the contribution of unobscured SFR as traced by the UV light.

predicted CCSN rate in Arp 299 of 1.59-1.91 $\mathrm{yr}^{-1}$, we estimate a missing fraction of up to $83_{-15}^{+9} \%$, taking into account both statistical errors and the uncertainty in the total CCSN rate. We adopt these values as the missing fractions of CCSNe in local LIRGs (see Table 8).

\section{MISSING SUPERNOVAE AS A FUNCTION OF REDSHIFT}

From observations of local infrared luminous galaxies, we know that a large fraction of the SNe exploding in these galaxies are invisible to optical searches. For the local LIRG Arp 299, we have estimated that $\sim 83 \%$ of the SNe have probably been missed by optical observations. The local ULIRG Arp 220 has, similar to Arp 299, been monitored for SNe by several programs (e.g., Richmond et al. 1998; Leaman et al. 2011) without confirmed SN detection at optical or near-IR wavelengths despite the large CCSN rate inferred by interferometric radio observations (e.g., Lonsdale et al. 2006). Therefore, close to $100 \%$ of the SNe in such local ULIRGs are probably missed by optical searches. With an increased fraction of the total star formation occurring in LIRGs and ULIRGs at higher redshifts to at least $z \sim 2$ (Le Floc'h et al. 2005; Magnelli et al. 2009, 2011), it is expected that the fraction of SNe missed should also increase with time.

Using the missing fractions derived from local LIRGs and ULIRGs, however, is complicated by the fact that they do not have the same properties as their non-local counterparts. In fact, high-redshift ULIRGs seem to be more similar to local LIRGs than to Arp 220, showing more extended regions of star formation (e.g., Rujopakarn et al. 2011). While local ULIRGs have often a single nucleus and are in an advanced stage of merging (Veilleux et al. 2002), Kartaltepe et al. (2012) showed that high-redshift U/LIRGs show a wide range of morphological types including mergers, interactions, pure spheroids, and noninteracting disks. Furthermore, Kartaltepe et al. found that in total $\sim 43 \%$ of their high-redshift $(z>1.5)$ sample of LIRGs and ULIRGs are starbursting, defined as having a specific SFR a factor 3 higher than the star-forming main sequence (Elbaz et al. 2011).

Alonso-Herrero et al. (2009) have demonstrated a good match between the PAH features of Arp 299 in their integrated midIR spectrum of the whole system and in the spectra of high- $z$ ULIRGs. For the integrated spectrum of Arp 299 they used a large extraction aperture including all the nuclei and also a significant amount of circumnuclear star formation in regions between IC 694 and NGC 3690. The level of obscuration in Arp 299 appears to be similar to that in M 82, submillimeter galaxies, and the high- $z$ ULIRGs in the sample of Farrah et al. (2008), but it is lower than that in local ULIRGs. If the majority of high-redshift galaxies have lower levels of obscuration than Arp 299 (as indicated by the ratio of total IR to $8 \mu$ m luminosity), we should assume a lower missing SN fraction at high redshift for that fraction of the galaxies. Using these considerations, we derive three models for the expected missing fraction of CCSNe as a function of redshift.

The missing fraction of SNe in high-redshift U/LIRGs is highly uncertain. For the main-sequence (i.e., non-starburst) galaxies, little is known about the extinction, although they have been found to be disk-like and not as compact as their local U/LIRG counterparts (Kartaltepe et al. 2012). But, given that they are luminous in the mid- and far-IR, they contain substantially more gas and dust than local disk galaxies. It therefore seems likely that the extinction experienced by SNe in these galaxies is higher on average than in the local population of normal galaxies. Furthermore, Reddy et al. (2012) found that roughly $80 \%$ of the star formation in typical star-forming galaxies at $z \sim 2$ is obscured (at UV wavelengths) by dust. We assume that the level of obscuration in these systems is roughly similar to that of the circumnuclear regions in Arp 299 and thus adopt a $37 \%$ missing fraction of $\mathrm{SNe}$ in these galaxies.

To calculate the total missing fraction as a function of redshift, we use the relative contributions to the cosmic star formation density of normal galaxies (defined as galaxies with $\left.L_{\mathrm{IR}}<10^{11} L_{\odot}\right)$, LIRGs, and ULIRGs from Magnelli et al. (2011). We then assume that SNe either explode in normal (i.e., with low-to-moderate IR luminosity) galaxies, starburst U/LIRGs, or main-sequence (high-redshift) U/LIRGs. Following Magnelli et al. (2011), the total SFR density at each redshift is defined as the sum of the unobscured SFR density traced by the UV light (accounting for $\sim 20 \%$ of the total SFR density) and obscured SFR density traced by the IR light. We assume a zero missing fraction for the $\mathrm{SNe}$ originating from the unobscured star formation. However, we note that our estimated missing fraction in normal galaxies can include $\mathrm{SNe}$ originating from both obscured (e.g., SNe 2002hh in NGC 6946) and unobscured star formation (e.g., SN 2008jb in a dwarf irregular host galaxy with a metallicity similar to the Small Magellanic Cloud). We have therefore corrected this missing SN fraction to correspond only to the obscured star formation in normal galaxies. For this correction we adopted a local fraction of unobscured star formation in normal galaxies of $17 \%$, which we obtained by combining the unobscured UV star formation from Schiminovich et al. (2005) with the IR star formation results of Magnelli et al. (2011) and assuming the contribution of the unobscured UV light to the bolometric luminosity of U/LIRGs to be negligible. Our estimated missing fractions in normal galaxies (both observed and corrected) and in U/LIRGs are summarized in Table 8. In the following we adopt the results of Kartaltepe et al. (2012) according to which about $42.6 \%$ of the U/LIRGs at $z \sim 2$ are starbursting. Since there is no strong redshift dependence in the Kartaltepe data at $1.5<z<2.5$, we assume that this value is valid at $z=1.5$, and interpolate the fraction of starbursting U/LIRGs between $z=0(100 \%)$ and $z=1.5$ (42.6\%) assuming a simple linear evolution. At $z>1.5$, we keep the fraction constant.

The effects of host galaxy extinction on the detectability of $\mathrm{SNe}$ are also expected to increase with redshift since, in general, shorter rest-frame wavelengths are observed at higher redshifts. However, we note that current SN searches are often optimized for detecting SNe at rest-frame $B$ and $V$ bands, since the SN SED 
Table 9

Models for the Missing SN Fraction

\begin{tabular}{lcccc}
\hline \hline Model & Normal & \multicolumn{3}{c}{ U/LIRGs } \\
\cline { 3 - 5 } & & Local & Starburst & Non-starburst \\
\hline Nominal & $19 \%$ & $83 \%$ & $83 \%$ & $37 \%$ \\
Low & $7 \%$ & $83 \%$ & $83 \%$ & $19 \%$ \\
High & $43 \%$ & $83 \%$ & $83 \%$ & $83 \%$ \\
\hline
\end{tabular}

Note. The missing SN fractions in normal galaxies, local U/LIRGs, and in U/LIRGs at $z>0$ which are starbursting and non-starbursting.

peaks in this range. The uncertain nature of $\mathrm{U} / \mathrm{LIRG}$ at high- $z$ currently far outweighs any wavelength-dependent effects on our predictions for the missing $\mathrm{SN}$ fractions and we do not attempt to account for these. Therefore, our predictions are only valid for rest-frame optical $\mathrm{SN}$ searches.

Bothwell et al. (2011) derived the local SFR distribution function and found $\sim 20 \%$ of the star formation occurring in starbursts defined as systems forming stars at $\geqslant 10 M_{\odot} \mathrm{yr}^{-1}$. They also studied the contribution of U/LIRGs to the local SFR volume density and found a value of $\sim 10 \%$. Therefore, $\sim 10 \%$ of the local SFR can be assumed to occur in starbursts with $L_{\mathrm{IR}}<10^{11} L_{\odot}$ (e.g., M 82 and NGC 4038/9). However, the missing fraction of $\mathrm{SNe}$ in such galaxies is not well constrained. In addition, we know little about their evolution as a function of redshift. Given these substantial uncertainties we decided not to consider starburst galaxies with $L_{\mathrm{IR}}<10^{11} L_{\odot}$ separately and instead assume that these are included in our missing SN fraction estimated for the normal $L_{\mathrm{IR}}<10^{11} L_{\odot}$ galaxies. We expect that this decision does not significantly affect our final results. For example, if adopting a similar missing $\mathrm{SN}$ fraction for the starburst galaxies as found for the circumnuclear regions of Arp 299 instead of the value found for normal galaxies, we find that the local missing SN fraction would increase only by two percentage points, which is negligible compared to the other uncertainties (see below).

Based on these assumptions, we derive three different models for the missing fraction and its dependence on redshift. We call these Nominal, Low, and High missing fraction models (for a summary, see Table 9).

Nominal model. The nominal model is based on the assumption that the fraction of the U/LIRGs that are compact and starbursting decreases with redshift, following the results from Kartaltepe et al. We assume that Arp 299 represents local U/LIRGs. At high redshift we assume that Arp 299 only represents the U/LIRGs that are starbursting, i.e., lie more than three times above the specific star formation main-sequence locus. To calculate the missing fraction, we assume that all starbursting U/LIRGs have a missing fraction of $83 \%$. For the non starbursting U/LIRGs, we assume that the missing fraction can be represented by the value estimated for the circumnuclear regions of Arp 299, i.e., 37\% of missing SNe. For normal galaxies, we assume a $19 \%$ missing fraction.

Low missing fraction. Again, we assume that Arp 299 represents local U/LIRGs and at high redshift it only represents the U/LIRGs that are starbursting. To calculate the missing fraction, we assume that all starbursting U/LIRGs have a missing fraction of $83 \%$, while the $z>0 \mathrm{U} /$ LIRGs that are not starbursting have the same missing fraction as the normal galaxies (i.e., 19\%). For the low missing fraction case, we further assume that the remaining normal galaxies have a missing fraction given by the $1 \sigma$ lower limit of our estimate, i.e., $7 \%$.

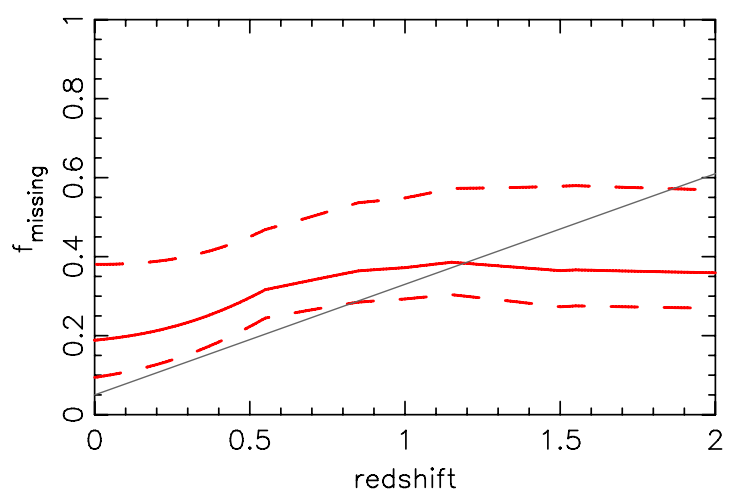

Figure 5. Fraction of SNe missed by rest-frame optical searches as a function of redshift. Red lines show our best (Nominal) estimate together with Low and High missing fraction models as dashed lines. The solid black line is the missing fraction from Mannucci et al. (2007).

(A color version of this figure is available in the online journal.)

Table 10

Missing SN fraction

\begin{tabular}{lccc|ccc}
\hline \hline Redshift & Nominal & Low & High & $f$ (normal) & $f$ (LIRG) & $f$ (ULIRG) \\
\hline 0.0 & 0.189 & 0.094 & 0.381 & 0.146 & 0.040 & 0.003 \\
0.1 & 0.198 & 0.108 & 0.382 & 0.139 & 0.056 & 0.004 \\
0.2 & 0.213 & 0.127 & 0.388 & 0.131 & 0.077 & 0.005 \\
0.3 & 0.233 & 0.152 & 0.401 & 0.121 & 0.104 & 0.008 \\
0.4 & 0.261 & 0.184 & 0.421 & 0.111 & 0.140 & 0.010 \\
0.5 & 0.296 & 0.223 & 0.451 & 0.099 & 0.183 & 0.014 \\
0.6 & 0.325 & 0.252 & 0.479 & 0.092 & 0.215 & 0.018 \\
0.7 & 0.341 & 0.266 & 0.502 & 0.089 & 0.231 & 0.021 \\
0.8 & 0.356 & 0.279 & 0.525 & 0.086 & 0.246 & 0.024 \\
0.9 & 0.367 & 0.288 & 0.540 & 0.081 & 0.257 & 0.030 \\
1.0 & 0.372 & 0.293 & 0.549 & 0.073 & 0.261 & 0.038 \\
1.1 & 0.381 & 0.300 & 0.564 & 0.066 & 0.267 & 0.048 \\
1.2 & 0.383 & 0.299 & 0.573 & 0.061 & 0.264 & 0.058 \\
1.3 & 0.377 & 0.291 & 0.574 & 0.058 & 0.254 & 0.065 \\
1.4 & 0.371 & 0.282 & 0.576 & 0.055 & 0.243 & 0.073 \\
1.5 & 0.365 & 0.273 & 0.578 & 0.051 & 0.232 & 0.081 \\
1.6 & 0.366 & 0.275 & 0.578 & 0.050 & 0.229 & 0.087 \\
1.7 & 0.364 & 0.273 & 0.576 & 0.050 & 0.229 & 0.085 \\
1.8 & 0.362 & 0.272 & 0.573 & 0.050 & 0.228 & 0.084 \\
1.9 & 0.361 & 0.271 & 0.571 & 0.050 & 0.228 & 0.083 \\
2.0 & 0.359 & 0.269 & 0.569 & 0.050 & 0.228 & 0.082 \\
\hline
\end{tabular}

Notes. The missing SN fractions calculated for different redshift bins following the assumptions from Tables 8 and 9. Columns 5-7 show the contribution to the nominal missing fraction from normal galaxies, LIRGs, and ULIRGs, respectively.

High missing fraction. We assume that all ULIRGs and LIRGs can be represented by Arp 299 at all redshifts, having a missing SN fraction of $83 \%$. This should be an upper limit since the SFR of Arp 299 is dominated by the compact and highly obscured nuclei A and B1+B2 whereas the U/LIRG population at high redshifts is dominated by galaxies forming stars in the "normal" main-sequence mode probably more similar to the circumnuclear star formation in Arp 299. For this model, we also assume an upper limit for the missing fraction in normal galaxies by using the $1 \sigma$ upper limit, i.e., $43 \%$ of the $\mathrm{SNe}$ missing.

Using these assumptions and the evolution of the SFR and its contribution from normal galaxies, LIRGs, and ULIRGs taken from Magnelli et al. (2011), we calculate how the missing fraction of CCSNe evolves with redshift (see Table 10). In Figure 5, we plot the nominal model as a solid line, while the 
Table 11

Parametrization of the missing SN fraction

\begin{tabular}{lrc}
\hline \hline Redshift & $\mathrm{k}$ & $\mathrm{m}$ \\
\hline$z<0.25$ & 0.135 & 0.188 \\
$0.25<z<0.55$ & 0.314 & 0.144 \\
$0.55<z<1.15$ & 0.116 & 0.253 \\
$1.15<z<1.55$ & -0.049 & 0.442 \\
$1.55<z<2.00$ & -0.016 & 0.392 \\
\hline
\end{tabular}

Note. A straight line parametrization for the nominal missing SN fraction: $f_{\text {missing }}=k \times z+m$.

Low and High missing fraction models are shown with dashed lines. We also compare these with the prediction from Mannucci et al. (2007). The main differences between the two models is the higher missing SN fraction locally in our case, which is a consequence of the relatively high fraction (2 out of 13) of local CCSNe that showed a high amount of dust extinction even in moderately inclined host galaxies. We also note that our missing $\mathrm{SN}$ fraction model levels out at $z \gtrsim 1.2$, reflecting the fact that we assume that high-redshift U/LIRGs are not as compact as the local ones, e.g., Arp 299, and therefore have a lower fraction of missing CCSNe.

Our derived missing SN fraction can be well represented by straight lines within the different redshift bins according to

$$
f_{\text {missing }}=k \times z+m \text {, }
$$

where the values for $[k, m]$ are listed in Table 11 for the nominal model. To correct the derived CCSN rates for the missing fraction of $\mathrm{SNe}$ hidden in highly extinguished environments, the results have to be multiplied by a de-bias factor given by

$$
f_{\text {de-bias }}=\frac{1}{1-f_{\text {missing }}} .
$$

\section{DISCUSSION AND SUMMARY}

The SN events with high extinctions can have an important impact for SN statistics when estimating the complete CCSN rates including the optically obscured SNe. This is essential when using CCSNe as probes of the SFR at both low- and high- $z$ (e.g., Cappellaro et al. 1999; Dahlen et al. 2004, 2012; Botticella et al. 2008; Melinder et al. 2012) with the aim of providing a new independent measurement of the cosmic star formation history. Furthermore, accurate determination of the complete CCSN rates will be crucial for comparison with the diffuse SN neutrino background in the future (e.g., Lien et al. 2010). We have shown that a substantial fraction of CCSNe have remained undetected by current optical SN searches due to obscuration by large amounts of dust in their host galaxies. We find that there should be missing CCSNe in highly extinguished environments in both normal host galaxies (even with moderate inclination) by $\sim 5 \%-36 \%$ and in highly dust-enshrouded environments in U/LIRGs by up to $\sim 70 \%-90 \%$. We note that our estimated missing SN fraction in normal galaxies is also consistent with the recent findings of Michałowski et al. (2012) for their sample of $z \lesssim 1$ gamma-ray burst host galaxies. For a volume-limited rest-frame optical SN survey we find the missing SN fraction to increase from its average local value of $\sim 19 \%$ to $\sim 38 \%$ at $z \sim$ 1.2 and then stay roughly constant up to $z=2$.

Using a local sample of CCSNe discovered during the last $12 \mathrm{yr}$, we find a lower limit for the local CCSN rate of
$1.5_{-0.3}^{+0.4} \times 10^{-4} \mathrm{yr}^{-1} \mathrm{Mpc}^{-3}$ within the 6-15 Mpc volume, which is consistent with the CCSN rate estimate within $11 \mathrm{Mpc}$ from Botticella et al. (2012). Our estimated CCSN rate is significantly higher than the volumetric CCSN rate from the LOSS $(\mathrm{Li}$ et al. 2011a) of $(0.84 \pm 0.18) \times 10^{-4} \mathrm{yr}^{-1} \mathrm{Mpc}^{-3}$ (scaled to correspond to $H_{0}=70 \mathrm{~km} \mathrm{~s}^{-1} \mathrm{Mpc}^{-1}$ ). If applying our newly derived missing $\mathrm{SN}$ fraction correction, then the volumetric rate of LOSS would become $(1.04 \pm 0.22) \times 10^{-4} \mathrm{yr}^{-1} \mathrm{Mpc}^{-3}$ or $(1.35 \pm 0.29) \times 10^{-4} \mathrm{yr}^{-1} \mathrm{Mpc}^{-3}$ if adopting the "high missing fraction" model. Therefore, within the uncertainties in the missing SN fraction correction, this is consistent with our CCSN rate estimate within 6-15 Mpc. We note that LOSS made use of their observed luminosity functions of SNe to compensate for the effects of host galaxy extinction for their derived SN rates. Their results indicated that the line-of-sight extinctions toward SNe in the highly inclined galaxies were not significantly higher than in the less inclined systems. However, they noted that this could still be a consequence of a small number of SNe. However, there are also a number of other possible contributing factors to the difference between the CCSN rate estimates, including the possibility of LOSS missing a larger fraction of the intrinsically faint events than our $15 \mathrm{Mpc}$ sample. Also, we note that the volumetric CCSN rate of LOSS has been obtained by multiplying their estimated rates in units of galaxy $K$-band luminosities (SNuK) with the local $K$-band luminosity density introducing additional uncertainties.

Our CCSN rate within 6-15 Mpc may also be elevated by cosmic variance. Assuming a Salpeter IMF between 0.1 and $125 M_{\odot}$ and CCSN progenitor masses between 8 and $50 M_{\odot}$, we find that our CCSN rate corresponds to an SFR $0.021_{-0.005}^{+0.006} M_{\odot} \mathrm{yr}^{-1} \mathrm{Mpc}^{-3}$. This is very similar to the local rate $0.019 M_{\odot} \mathrm{yr}^{-1} \mathrm{Mpc}^{-3}$ of Horiuchi et al. (2011), where the latter rate is given for the cosmology adopted here. The SFR within 11 Mpc based on the HUGS program (Kennicutt et al. 2008; Bothwell et al. 2011) should, however, be similarly affected by cosmic variance, allowing a more direct comparison between rates. The SFR derived in Bothwell et al. $0.023_{-0.002}^{+0.002} M_{\odot}$ $\mathrm{yr}^{-1} \mathrm{Mpc}^{-3}$ (given for our adopted cosmology) is consistent with our rate within the error bars. This is also similar to the rate of Magnelli et al. (2009). We therefore conclude that our rate is consistent with what is expected from the SFR and there is no need to correct for SNe missed in our nearby sample. Horiuchi et al. (2011) and Melinder et al. (2012) discuss the choice of the IMF, and they show that the IMF dependence is mostly canceled out as long as the same IMF is used when originally scaling from the massive star SFR to the total SFR and when converting between the CCSN rate and the SFR. Here we have adopted the Salpeter IMF, which has also been used for deriving the SFRs. We also note that calculating the CCSN rate in the very nearby universe within a distance of $<6 \mathrm{Mpc}$, we find a significant increase in the rate by a factor $\sim 5$ compared to the rate found within 6-15 Mpc, caused by a few galaxies that have each hosted multiple SNe. This further supports the suggestion of a significant local overdensity in the SFR within $\sim 10 \mathrm{Mpc}$ (e.g., Karachentsev et al. 2004).

Horiuchi et al. (2011) note that local and low-redshift CCSN rates published before 2011 are lower compared to those expected from the SFR by a factor $\sim 2$ at a $2 \sigma$ confidence. As a solution to this discrepancy, they suggested that there could be a population of faint $\operatorname{CCSNe}(M \sim-15)$ that are typically missed by SN surveys or that there could be a population of SNe hidden by dust, or a combination of these two effects. Using their sample of CCSNe a distance of within $11 \mathrm{Mpc}$, Botticella 
et al. (2012) did a detailed comparison with the local SFRs and found their CCSN rate to be consistent with that expected from the SFR derived from far-UV luminosities and higher than expected based on $\mathrm{H} \alpha$ luminosities. In our CCSN sample within $12 \mathrm{Mpc}$, there are no CCSNe fainter than $M \sim-15$ and roughly $20 \%$ fainter than $M \sim-15.5$. These intrinsically faint events are more likely to be missed in $\mathrm{SN}$ searches over a larger volume compared to our $<12 \mathrm{Mpc}$ sample and could therefore lead to underestimates of the CCSN rate. Even if such events at the peak would be above the magnitude limit of the survey, the time on the light curve they spend above the limiting magnitude of the search is shorter for this population and unless accounted for will lead to an underestimate of the rates.

Using our local sample of CCSNe, for which we were able to include $\mathrm{SNe}$ both with high extinctions and faint intrinsic magnitudes, we do not find any discrepancy with the expectations from the SFRs, even when taking the cosmic variance into account. Looking at the fraction of SNe missed in highly extinguished environments, we have found locally $f_{\text {missing }}=19_{-10}^{+19} \%$, corresponding to a de-bias factor of $\sim 1.1-1.6$. This is smaller than the factor $\sim 2$ suggested by Horiuchi et al. (2011) but together with a realistic fraction of intrinsically faint events can account for the apparent discrepancy between some of the previous local CCSN rate estimates and the expectations from the SFRs. The effects of extinction correction on the CCSN rates at higher redshifts are presented and discussed thoroughly in Melinder et al. (2012) and Dahlen et al. (2012). We therefore conclude that correcting for the CCSNe missed due to very high dust extinctions in their host galaxies is crucial for deriving accurate CCSN rates. Taking these effects into account should lead to CCSN rates that are consistent with those expected from the SFRs.

We thank the anonymous referee for several useful suggestions, Enrico Cappellaro and Maria Teresa Botticella for comments in the manuscript, and Nancy Elias de la Rosa and Chris Dudley for helpful discussions. We thank Benjamin Magnelli for providing data over the redshift evolution of the IR luminosity densities. S.M. and E.K. acknowledge financial support from the Academy of Finland (project: 8120503). A.A.H. thanks the Spanish Plan Nacional through grant AYA201021161-C02-01 and the Universidad de Cantabria through the Augusto G. Linares program for financial support. M.A.P.T. acknowledges support by the Spanish MICINN through grant AYA2009-13036-CO2-01, partially funded by FEDER funds. This research has also been partially funded by the Autonomic Government of Andalusia under grants P08-TIC-4075 and TIC126. This work is based on observations obtained at the Gemini Observatory, which is operated by the Association of Universities for Research in Astronomy, Inc., under a cooperative agreement with the NSF on behalf of the Gemini partnership: the National Science Foundation (United States); the Science and Technology Facilities Council (United Kingdom); the National Research Council (Canada); CONICYT (Chile); the Australian Research Council (Australia); Ministrio da Cincia, Tecnologia e Inovao (Brazil); and Ministerio de Ciencia, Tecnologa e Innovacin Productiva (Argentina). We acknowledge the usage of the HyperLeda database (http://leda.univ-lyon1.fr).

\section{REFERENCES}

Alonso-Herrero, A., Pereira-Santaella, M., Rieke, G. H., \& Rigopoulou, D. 2012, ApJ, 744, 2

Alonso-Herrero, A., Rieke, G. H., Colina, L., et al. 2009, ApJ, 697, 660
Alonso-Herrero, A., Rieke, G. H., Rieke, M. J., \& Scoville, N. Z. 2000, ApJ, 532,845

Anderson, J. P., Habergham, S. M., \& James, P. A. 2011, MNRAS, 416, 567

Anderson, J. P., Habergham, S. M., James, P. A., \& Hamuy, M. 2012, MNRAS, 424,1372

Arcavi, I., Gal-Yam, A., Yaron, O., et al. 2011, ApJ, 742, L18

Barbon, R., Buondí, V., Cappellaro, E., \& Turatto, M. 1999, A\&AS, 139, 531

Bazin, G., Palanque-Delabrouille, N., Rich, J., et al. 2009, A\&A, 499, 653

Berger, E., Soderberg, A. M., Chevalier, R. A., et al. 2009, ApJ, 699, 1850

Beswick, R. J., Pérez-Torres, M. A., Mattila, S., et al. 2010, ATel, 2432, 1

Blondin, S., \& Tonry, J. L. 2007, ApJ, 666, 1024

Bond, H. E., Bedin, L. R., Bonanos, A. Z., et al. 2009, ApJ, 695, L154

Bondi, M., Pérez-Torres, M. A., Herrero-Illana, R., \& Alberdi, A. 2012, A\&A, 539, A134

Bothwell, M. S., Kenicutt, R. C., Johnson, B. D., et al. 2011, MNRAS, 415, 1815

Botticella, M. T., Pastorello, A., Smartt, S. J., et al. 2009, MNRAS, 398, 1041 Botticella, M. T., Riello, M., Cappellaro, E., et al. 2008, A\&A, 479, 49

Botticella, M. T., Smartt, S. J., Kennicutt, R. C., et al. 2012, A\&A, 537, A132 Branch, D., Benetti, S., Kasen, D., et al. 2002, ApJ, 566, 1005

Brunthaler, A., Martí-Vidal, I., Menten, K. M., et al. 2010, A\&A, 516, A27

Bruzual, G., \& Charlot, S. 2003, MNRAS, 344, 1000

Cappellaro, E., Evans, R., \& Turatto, M. 1999, A\&A, 351, 459

Caputi, K. I., Lagache, G., Yan, L., et al. 2007, ApJ, 660, 97

Cardelli, J. A., Clayton, G. C., \& Mathis, J. S. 1989, ApJ, 345, 245

Charmandaris, V., Stacey, G. J., \& Gull, G. 2002, ApJ, 571, 282

Chornock, R., Filippenko, A. V., Li, W., et al. 2011, ApJ, 739, 41

Dahlen, T., Strolger, L.-G., Riess, A. G., et al. 2004, ApJ, 613, 189

Dahlen, T., Strolger, L.-G., Riess, A. G., et al. 2012, ApJ, in press (arXiv:1208.0342)

Dudley, C. C., Fischer, J., \& Li, W. 2008, IAU Circ., 8992, 1

Efstathiou, A., \& Rowan-Robinson, M. 1995, MNRAS, 273, 649

Efstathiou, A., Rowan-Robinson, M., \& Siebenmorgen, R. 2000, MNRAS, 313 , 734

Efstathiou, A., \& Siebenmorgen, R. 2009, A\&A, 502, 541

Elbaz, D., Dickinson, M., Hwang, H. S., et al. 2011, A\&A, 533, A119

Elias-Rosa, N., Van Dyk, S. D., Li, W., et al. 2011, ApJ, 742, 6

Elmhamdi, A., Danziger, I. J., Branch, D., et al. 2006, A\&A, 450, 305

Farrah, D., Afonso, J., Efstathiou, A., et al. 2003, MNRAS, 343, 585

Farrah, D., Lonsdale, C. J., Weedman, D. W., et al. 2008, ApJ, 677, 957

Freedman, W. L., Madore, B. F., Gibson, B. K., et al. 2001, ApJ, 553, 47

García-Marín, M., Colina, L., Arribas, S., Alonso-Herrero, A., \& Mediavilla, E. 2006, ApJ, 650, 850

Gehrels, N. 1986, ApJ, 303, 336

Gieren, W., Pietrzyński, G., Soszyński, I., et al. 2005, ApJ, 628, 695

Graur, O., Poznanski, D., Maoz, D., et al. 2011, MNRAS, 417, 916

Grogin, N. A., Kocevski, D. D., Faber, S. M., et al. 2011, ApJS, 197, 35

Grossan, B., Spillar, E., Tripp, R., et al. 1999, AJ, 118, 705

Harutyunyan, A. H., Pfahler, P., Pastorello, A., et al. 2008, A\&A, 488, 383

Heckman, T. M. 1998, in ASP Conf. Proc. 148, Origins, ed. C. E. Woodward,

J. M. Shull, \& H. A. Thronson (San Francisco, CA: ASP), 127

Hendry, M. A., Smartt, S. J., Maund, J. R., et al. 2005, MNRAS, 359, 906

Herrero-Illana, R., Pérez-Torres, M. Á., \& Alberdi, A. 2012, A\&A, 540, L5

Hook, I., Jørgensen, I., Allington-Smith, J. R., et al. 2004, PASP, 116, 425

Horiuchi, S., Beacom, J. F., Kochanek, C. S., et al. 2011, ApJ, 738, 154

Hunter, D. J., Valenti, S., Kotak, R., et al. 2009, A\&A, 508, 371

Jha, S., Garnavich, P., Challis, P., et al. 1999, IAU Circ., 7089, 2

Kankare, E., Mattila, S., Ryder, S., et al. 2008, ApJ, 689, L97

Kankare, E., Mattila, S., Ryder, S., et al. 2012, ApJ, 744, L19

Karachentsev, I. D., Karachentseva, V. E., Huchtmeier, W. K., \& Makarov, D. I. 2004, AJ, 127, 2031

Karachentsev, I. D., Tully, R. B., Dolphin, A., et al. 2007, AJ, 133, 504

Kartaltepe, J. S., Dickinson, M., Alexander, D. M., et al. 2012, ApJ, in press (arXiv:1110.4057)

Kashi, A., Frankowski, A., \& Soker, N. 2010, ApJ, 709, L11

Kennicutt, R. C., Jr., Lee, J. C., Funes, S. J., et al. 2008, ApJS, 178, 247

Kochanek, C. S. 2011, ApJ, 741, 37

Krisciunas, K., Suntzeff, N. B., Candia, P., et al. 2003, AJ, 125, 166

Leaman, J., Li, W., Chornock, R., \& Filippenko, A. V. 2011, MNRAS, 412, 1419

Le Floc'h, E., Papovich, C., Dole, H., et al. 2005, ApJ, 632, 169

Leonard, D. C., \& Cenko, S. B. 2005, ATel, 431, 1

Li, W., Chornock, R., Leaman, J., et al. 2011a, MNRAS, 412, 1473

Li, W., Leaman, J., Chornock, R., et al. 2011b, MNRAS, 412, 1441

Li, W.-D., Li, C., Wan, Z., et al. 1998, IAU Circ., 6830, 1

Lien, A., Fields, D. B., \& Beacom, J. F. 2010, Physical Review D, 81, 083001 
Lonsdale, C. J., Diamond, P. J., Thrall, H., Smith, H. E., \& Lonsdale, C. J. 2006, ApJ, 647, 185

Magnelli, B., Elbaz, D., Chary, R. R., et al. 2009, A\&A, 496, 57

Magnelli, B., Elbaz, D., Chary, R. R., et al. 2011, A\&A, 528, A35

Maiolino, R., Vanzi, L., Mannucci, F., et al. 2002, A\&A, 389, 84

Mannucci, F., Della Valle, M., \& Panagia, N. 2007, MNRAS, 377, 1229

Mannucci, F., Maiolino, R., Cresci, G., et al. 2003, A\&A, 401, 519

Mattila, S., Fraser, M., Smartt, S. J., et al. 2012, MNRAS, submitted (arXiv:1207.1889)

Mattila, S., Greimel, R., Gerardy, C., et al. 2005a, IAU Circ., 8473, 1

Mattila, S., \& Kankare, E. 2010, CBET, 2145, 1

Mattila, S., Kankare, E., Datson, J., \& Pastorello, A. 2010, CBET, 2149, 1

Mattila, S., Lundqvist, P., Sollerman, J., et al. 2005b, A\&A, 443, 649

Mattila, S., \& Meikle, W. P. S. 2001, MNRAS, 324, 325

Mattila, S., Meikle, W. P. S., \& Greimel, R. 2004, New Astron. Rev., 48, 595

Mattila, S., Väisänen, P., Farrah, D., et al. 2007, ApJ, 659, L9

Maund, J. R., Smartt, S. J., Kudritzki, R.-P., et al. 2006, MNRAS, 369, 390

Melinder, J., Dahlen, T., Mencía-Trinchant, L., et al. 2011, A\&A, 532, A29

Melinder, J., Dahlen, T., Mencía-Trinchant, L., et al. 2012, A\&A, in press (arXiv:1206.6897)

Michałowski, M. J., Kamble, A., Hjorth, J., et al. 2012, ApJ, in press (arXiv:1205.4239)

Modjaz, M., Kirshner, R., Challis, P., \& Berlind, P. 2005, IAU Circ., 8475, 2

Monard, L. A. G., Milisavljevic, D., Fesen, R., et al. 2011, CBET, 2946, 1

Neff, S. G., Ulvestad, J. S., \& Teng, S. H. 2004, ApJ, 611, 186

Newton, J., Puckett, T., \& Orff, T. 2010, CBET, 2144, 2

Pastorello, A., Botticella, M. T., Trundle, C., et al. 2010, MNRAS, 408, 181

Paturel, G., Petit, C., Prugniel, P., et al. 2003, A\&A, 412, 45

Pereyra, A., Magalhães, A. M., Rodrigues, C. V., et al. 2006, A\&A, 454, 827

Pérez-González, P. G., Rieke, G. H., Egami, E., et al. 2005, ApJ, 630, 82

Pérez-Torres, M. A., Romero-Cañizales, C., Alberdi, A., \& Polatidis, A. 2009, A\&A, 507, L17

Pietrzyński, G., Gieren, W., Hamuy, M., et al. 2010, AJ, 140, 1475

Pozzo, M., Meikle, W. P. S., Rayner, J. T., et al. 2006, MNRAS, 368, 1169

Prieto, J. L., Lee, J. C., Drake, A. J., et al. 2012, ApJ, 745, 70
Pumo, M. L., Turatto, M., Botticella, M. T., et al. 2009, ApJ, 705, L138

Reddy, N., Dickinson, M., Elbaz, D., et al. 2012, ApJ, 744, 154

Richmond, M. W., Filippenko, A. V., \& Galisky, J. 1998, PASP, 110, 553

Riello, M., \& Patat, F. 2005, MNRAS, 362, 671

Rodney, S. A., Riess, A. G., Dahlen, T., et al. 2012, ApJ, 746, 5

Romero-Cañizales, C., Mattila, S., Alberdi, A., et al. 2011, MNRAS, 415, 2688

Romero-Cañizales, C., Perez-Torres, M. A., Alberdi, A., et al. 2012, A\&A, 543, A72

Rowan-Robinson, M., \& Efstathiou, A. 2009, MNRAS, 399, 615

Rujopakarn, W., Rieke, G. H., Eisenstein, D. J., \& Juneau, S. 2011, ApJ, 726, 93

Sanders, D. B., Mazzarella, J. M., Kim, D.-C., Surace, J. A., \& Soifer, B. T. 2003, AJ, 126, 1607

Schiminovich, D., Ilbert, O., Arnouts, S., et al. 2005, ApJ, 619, L47

Schlegel, D. J., Finkbeiner, D. P., \& Davis, M. 1998, ApJ, 500, 525

Schmidt, B., \& Salvo, M. 2005, CBET, 122, 1

Silbermann, N. A., Harding, P., Madore, B. F., et al. 1996, ApJ, 470, 1

Silverman, J. M., Mazzali, P., Chornock, R., et al. 2009, PASP, 121, 689

Smartt, S. J., Eldridge, J. J., Crockett, R. M., \& Maund, J. R. 2009, MNRAS, 395, 1409

Smith, N., Ganeshalingam, M., Chornock, R., et al. 2009, ApJ, 697, L49

Smith, N., Li, W., Silverman, J. M., Ganeshalingam, M., \& Filippenko, A. V. 2011, MNRAS, 415, 773

Soifer, B. T., Neugebauer, G., Matthews, K., et al. 2001, AJ, 122, 1213

Solanes, J. M., Sanchis, T., Salvador-Solé, E., Giovanelli, R., \& Haynes, M. P. 2002, AJ, 124, 2440

Szczygiel, D. M., Prieto, J. L., Kochanek, C. S., et al. 2012, ApJ, 750, 77

Takada-Hidai, M., Aoki, W., \& Zhao, G. 2002, PASJ, 54, 899

Tsvetkov, D. Y. 1994, Astron. Lett., 20, 374

Ulvestad, J. S. 2009, AJ, 138, 1529

van Buren, D., Jarrett, T., Terebey, S., et al. 1994, IAU Circ., 5960, 2

Van Dyk, S. D., Davidge, T. J., Elias-Rosa, N., et al. 2012, AJ, 143, 19

Veilleux, S., Kim, D.-C., \& Sanders, D. B. 2002, ApJS, 143, 315

Wagner, R. M., Vrba, F. J., Henden, A. A., et al. 2004, PASP, 116, 326

Young, D. R., Smartt, S. J., Mattila, S., et al. 2008, A\&A, 489, 359 